\title{
A Cerebral Central Pattern Generator in Aplysia and Its Connections with Buccal Feeding Circuitry
}

\author{
Ray Perrins and Klaudiusz R. Weiss \\ Department of Physiology and Biophysics, Mount Sinai School of Medicine, Mount Sinai Medical Center, New York, \\ New York 10029-6574
}

\begin{abstract}
Different feeding-related behaviors in Aplysia require substantial variations in the coordination of movements of two separate body parts, the lips and buccal mass. The central pattern generators (CPGs) and motoneurons that control buccal mass movements reside largely in the buccal ganglion. It was previously thought that control of the cerebral neuronal circuitry and motoneurons that generate lip movements was coordinated directly by feedback from buccal interneurons. Here, we describe cerebral lip motoneuron $\mathrm{C} 15$, which drives rhythmic activity in the isolated cerebral ganglion. Other lip motoneurons are active during this program, so we define it as a cerebral motor program (CMP). The C15 in each cerebral hemiganglion drives the CMP in ipsilateral neurons only, suggesting there are independent CPGs in each hemiganglion. The cerebral and buccal CPGs interact at several points. For example, cerebralto-buccal interneurons (CBIs), which can drive the buccal CPG,
\end{abstract}

receive excitatory input when the cerebral CPG is active. Likewise, C15, which can drive the cerebral CPG, is excited when the buccal CPG is active. This excitation is simultaneous in both $\mathrm{C} 15 \mathrm{~s}$, coupling the activity in the two hemiganglionic cerebral CPGs. Therefore, there are independent cerebral and buccal CPGs, which can produce distinct rhythms, but which interact at several points. Furthermore, the connections between the cerebral and buccal CPGs alter during different forms of motor program. We suggest that such alterations in the interactions between these CPGs might contribute to the generation of the various forms of coordination of lip and buccal mass movements that are necessary during different feedingrelated behaviors.

Key words: Aplysia; feeding; central pattern generator; motor program; command neuron; coupled oscillators
Most animals need to alter the coordination of different body parts to produce a variety of behaviors. For example, the coordination of different limbs changes during various locomotor gaits. It has been proposed, in several such systems, that there is series of individual central pattern generators (CPGs), one for each limb or joint (vertebrates: Grillner and Wallen, 1985; Rossignol et al., 1993; invertebrates: Mulloney et al., 1993; Ryckebusch and Laurent, 1994). These CPGs could interact in distinct ways to produce the different motor patterns needed to generate different gaits or even separate behaviors such as scratching. It should be noted that this is a different situation than that for chains of oscillators, which are coupled to produce undulatory swimming behaviors, as has been proposed in the leech (Friesen and Pearce, 1993) and lamprey (Sigvardt, 1993). In these systems, individual oscillators are largely identical, whereas in the examples for walking described above, each limb or joint CPG may have quite different intrinsic properties.

Most progress in understanding the mechanisms of rearrangement of oscillatory networks has been accomplished in the crustacean stomatogastric nervous system (for review, see Dickinson and Moulins, 1992). In that system, four CPGs control body parts that are responsible for separate components of the ingestive behavior. Thus, although interactions do occur, the CPGs always

\footnotetext{
Received June 10, 1996; revised Aug. 2, 1996; accepted Aug. 9, 1996.

This work was supported by Human Frontiers Science Program Grant LT-561/95 and National Institutes of Health Grants GM 32009 and MH 50235. We thank Drs.

E. C. Cropper and C. G. Evans for comments on earlier versions of this manuscript. Correspondence should be addressed to Ray Perrins, Department of Physiology and Biophysics, Mount Sinai School of Medicine, Mount Sinai Medical Center, One Gustave L. Levy Place, New York, NY 10029-6574.

Copyright (C) 1996 Society for Neuroscience $0270-6474 / 96 / 167030-16 \$ 05.00 / 0$
}

produce rhythms with distinct cycle periods. In Aplysia, two body parts, the lips and radula, must be coordinated on a cycle-by-cycle basis during various feeding-related behaviors. In this paper, we show that, as postulated for locomotion, separate CPGs control each body part and that the interactions between these CPGs alter during different motor programs.

The consumatory phase of feeding in Aplysia consists of rhythmic movements of the radula, which are coordinated with appropriate opening and closing of the lips (Morton and Chiel, 1993a). The intrinsic buccal mass muscles that control the movement of the radula are innervated by motoneurons in the buccal ganglion (Cohen et al., 1978; Church and Lloyd, 1994). Firing of these motoneurons during feeding-related behaviors is controlled by CPGs, the neuronal circuitry for which appears to reside largely in the buccal ganglion (Weiss et al., 1982; Susswein and Byrne, 1988; Kirk, 1989). Motoneurons innervating lip muscles are located in the cerebral ganglion (Chiel et al., 1986; Rosen et al., 1989). It was previously considered likely that behaviorally appropriate output from these cerebral motoneurons was coordinated from the buccal ganglion. Each cerebral motoneuron was thought to be directly activated by a number of buccal-to-cerebral interneurons (BCIs) (Chiel et al., 1988; Teyke et al., 1993; Rosen et al., 1991a).

Here, we present evidence that each cerebral hemiganglion contains a CPG that can produce a cerebral motor program (CMP) in isolated cerebral ganglia. The cerebral CPGs provide an alternative means of activating lip motoneurons to the direct control by BCIs. We characterize neuron $\mathrm{C} 15$, which drives the CMP, describe basic features of the CMP, including synaptic drive to identified neurons, and demonstrate that different forms of 
behaviorally relevant motor programs are accompanied by changes in the interactions between cerebral and buccal CPGs.

\section{MATERIALS AND METHODS}

Experiments were performed at $15^{\circ} \mathrm{C}$ on Aplysia californica weighing 100-300 gm. Animals were anaesthetized by an injection of isotonic $\mathrm{MgCl}_{2}$ (50\% of body weight) into the body cavity. The cerebral ganglion was dissected out in isolation for some experiments and removed with the buccal ganglion attached for others (see Results for details). The ganglia were pinned onto a SYLGARD-coated dish at room temperature in artificial sea water (ASW). Cerebral ganglia were pinned ventralsurface-up and buccal ganglia rostral-surface-up. The connective tissue overlying the uppermost surface of the ganglia was surgically removed. The cerebral-pedal connective was rotated through $180^{\circ}$ and repinned to allow access to the lateral area of the E-cluster. Simultaneous intracellular recordings were made from up to four neurons using single or double-barreled microelectrodes filled with $2 \mathrm{~m}$ potassium acetate and beveled to a resistance ranging from 5 to $10 \mathrm{M} \Omega$, depending on the size of the cell to be penetrated. In preparations where the buccal ganglion was attached, buccal motor output was monitored using a polyethylene suction electrode placed over buccal nerve 2 (bn2) (see Morton and Chiel, 1993a) and connected to an AC amplifier. A recording or stimulating suction electrode was placed over the end of the severed cerebralto-buccal connective $(\mathrm{CBC})$ in experiments involving isolated cerebral ganglia.

To help identify previously described cells and to reveal the morphology of new ones, microelectrodes, beveled to a resistance of $10-15 \mathrm{M} \Omega$, were filled with a $3 \%$ solution of 5(6)-carboxyfluorescin in $0.1 \mathrm{M}$ potassium citrate, titrated to $\mathrm{pH} 8.0$ with $\mathrm{KOH}$ (see Rao et al., 1986). At the end of each experiment, previously described cells were identified on the basis of their morphology and known synaptic connections. In some experiments, polysynaptic pathways were suppressed using solutions containing elevated levels of divalent cations (with both $\mathrm{Ca}^{2+}$ and $\mathrm{Mg}^{2+}$ at three times the normal concentration) to raise neuronal firing thresholds.

To determine possible peripheral functions of $\mathrm{C} 15$, experiments were performed on preparations in which the cerebral ganglion was removed with the lips and anterior tentacles still attached by their peripheral nerves (cf. Rosen et al., 1979). The ganglion was pinned on an elevated SYLGARD platform, ventral-surface-uppermost, and desheathed as described above. The head structures were pinned, ventral-surfaceuppermost, to the bottom of the SYLGARD-coated dish in such a way as to reveal the inner portions of the lips and perioral zone (Rosen et al., 1982). The anterior aorta was cannulated and perfused with ASW.

Salines, all at $\mathrm{pH}$ 7.6, were composed as follows. ASW: NaCl, $460 \mathrm{~mm}$; $\mathrm{KCl}, 10 \mathrm{~mm} ; \mathrm{CaCl}_{2}, 11 \mathrm{~mm} ; \mathrm{MgCl}_{2}, 55 \mathrm{~mm} ; \mathrm{NaHCO}_{3}, 5 \mathrm{~mm}$. High divalent saline $\left(3 \times \mathrm{Mg}^{2+}, 3 \times \mathrm{Ca}^{2+}\right)$ : NaCl, $460 \mathrm{~mm} ; \mathrm{KCl}, 10 \mathrm{~mm} ; \mathrm{CaCl}_{2}, 33 \mathrm{~mm}$; $\mathrm{MgCl}_{2}, 165 \mathrm{~mm} ; \mathrm{NaHCO}_{3}, 5 \mathrm{~mm}$. For experiments in which chemical transmission was blocked, the $\mathrm{Ca}^{2+}$ was omitted and replaced with $10 \mathrm{~mm}$ $\mathrm{Co}^{2+}$. Experiments were performed at least six times, unless otherwise stated. Figures quoted are means \pm SE.

\section{RESULTS}

\section{Morphology and synaptic connections of C15}

We have discovered a neuron, $\mathrm{C} 15$, which was uniquely identified on the basis of its physiological and morphological properties. There was a single $\mathrm{C} 15$ in each half of the cerebral ganglion. It was a medium-sized oval neuron $(\sim 100-150 \mu \mathrm{m}$ in diameter) situated medially on the ventro-lateral surface of the E-cluster (JahanParwar and Fredman, 1976). The approximate positions of C15 and other cerebral neurons used in this study are shown schematically in Figure $1 A$. Injections of 5(6)-carboxyfluorescin into $\mathrm{C} 15$ revealed two peripheral axons, leaving the upper labial (ULAB) and anterior tentacular (AT) nerves (Fig. 1B). Dendritic arborisations were present in the E-cluster at the base of the $\mathrm{CBC}$ and in the M-cluster (Ono and McCaman, 1980). Processes were limited to the ipsilateral half of the ganglion.

In preparations in which the head structures were left connected to the cerebral ganglion, firing $\mathrm{C} 15$ at frequencies above 8 $\mathrm{Hz}$ produced a radial contraction in the tissue immediately adjacent to the anterior perioral zone of the ipsilateral inner lips. This resulted in an opening of the anterior portion of the jaws $(n=5)$. This contraction was observed before the occurrence of large IPSPs in C12, which would indicate the start CMP (see below). Firing $\mathrm{C} 15$ at these frequencies also readily produced the contraction while the whole preparation was bathed in solution containing $3 \times$ normal $\mathrm{Ca}^{2+}$ and $\mathrm{Mg}^{2+}$ (to suppress polysynaptic pathways), conditions under which the CMP was completely abolished. This means that the contraction was likely to be a direct effect of firing $\mathrm{C} 15$, which can therefore be described as a probable lip motoneuron. Mechanical or chemical (seaweed; Laver, Vega trading company, NY) stimuli applied to the lips, rhinophores, or tentacles had no effect on the membrane potential of $\mathrm{C} 15$, so we have no evidence for any sensory functions.

Two neighboring E-cluster neurons, C16 and C17, which are smaller and generally more rostral than $\mathrm{C} 15$, were also characterized in this study. C16 and C17 had physiological properties and synaptic inputs (see below) which were indistinguishable from $\mathrm{C} 15$. These neurons produced radial contractions in the tissue of the ipsilateral inner lips that persisted in high divalent solutions, like $\mathrm{C} 15$, so are also probable lip motoneurons. While C15 opened the anterior part of the jaws, $\mathrm{C} 16$ opened the medial region, and $\mathrm{C} 17$ the posterior. Firing all of these motoneurons together in the semi-intact preparation resulted in full opening of the ipsilateral jaws along their entire length. The motoneurons could also be distinguished from each other by the ability of C15 to reliably drive the CMP and, more important, by their morphology, revealed by injection of 5(6)-carboxyfluorescin. Not only were C16 and $\mathrm{C} 17$ generally smaller than $\mathrm{C} 15$, but they sent axons to the periphery via different combinations of nerves. $\mathrm{C} 16$ had axons in the AT and lower labial (LLAB) nerves and $\mathrm{C} 17$ had a single axon in the LLAB (Fig. 1C,D). These three motoneurons were electrically coupled to each other. Either a hyperpolarizing or a depolarizing current injected into $\mathrm{C} 15, \mathrm{C} 16$, or $\mathrm{C} 17$ resulted in a change of the same polarity but reduced amplitude in the membrane potential of the other two neurons (Fig. 2A). The coupling ratios (the voltage change in the postsynaptic neuron divided by the voltage change in the neuron into which current was injected) were between 0.1 and 0.2. Apparent coupling was higher when current was injected into $\mathrm{C} 15$ (Fig. $2 A$ ), possibly because of its larger size. Because $\mathrm{C} 2$ produced an IPSP in these neurons (see below), C15, C16, and C17 probably correspond to the Ea cluster of neurons of Chiel et al. (1986). These were described as inhibitory followers of $\mathrm{C} 2$, putative motoneurons for the lips that were in approximately the same position in the E-cluster.

C15 received chemical input from several cerebral and buccal neurons. The histaminergic mechanoafferent $\mathrm{C} 2$, which may play an important role in several feeding-related behaviors (Chiel et al., 1986), inhibited C15 (Fig. 2B) as well as C16 and C17. C15 also received input from several buccal-to-cerebral interneurons (BCIs: B18, B19, and B24), which are responsible for direct feedback to cerebral neurons during BMPs (Chiel et al., 1988; Rosen et al., 1990; Rosen et al., 1991a; Teyke et al., 1993). Spikes in B18 were followed one-for-one by fast EPSPs in C15, whereas firing in B19 produced a rapid inhibition in $\mathrm{C} 15$ followed by a slow excitation and B24 produced just an inhibitory response (Fig. $2 C-E)$. All of these responses persisted in salines containing $3 \times$ normal $\mathrm{Ca}^{2+}$ and $\mathrm{Mg}^{2+}$. Inputs from B18, B19, and B24 onto C16 and $\mathrm{C} 17$ were similar to those onto $\mathrm{C} 15$. There appeared to be no monosynaptic connections onto $\mathrm{C} 15, \mathrm{C} 16$, and $\mathrm{C} 17$ from another BCI, B20 (Teyke et al., 1993), although they all received excitatory input during the BMP driven by that cell. 


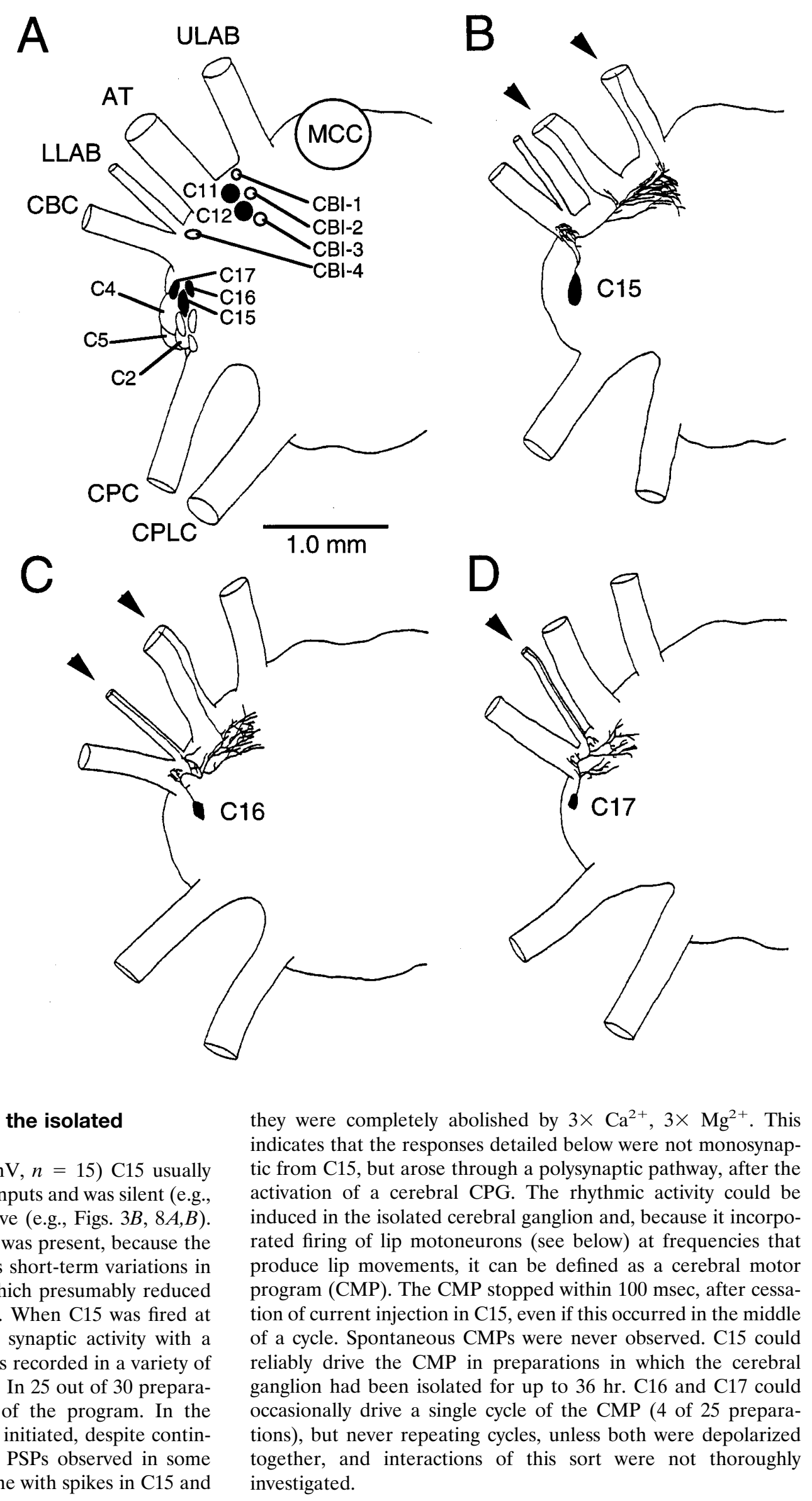

Figure 1. Position and morphology of cerebral motoneurons $\mathrm{C} 15, \mathrm{C} 16$, and C17. $A$, Schematic diagram of the ventral surface of a cerebral hemiganglion, showing the approximate positions of neurons in the E- and M-clusters used in this study. Filled cells indicate lip motoneurons. $B$, Drawing of the soma and central processes of $C 15$ filled by intracellular injection of 5(6)carboxyfluorescin and viewed with a fluorescence microscope. C15 had arborisations in the E- and M-clusters and peripheral axons in the ULAB and AT nerves. $C$, Morphology of $C 16$ was similar to that of C15, except it had axons in the AT and LLAB nerves. $D$, Morphology of $C 17$, which had a single axon in the LLAB. Arrowheads indicate nerves in which there are peripheral axons. $U L A B$, Upper labial nerve; $A T$, anterior tentacular nerve; $L L A B$, lower labial nerve; $C B C$, cerebralbuccal connective; $C P C$, cerebral-pedal connective; CPLC, cerebral-pleural connective.

\section{C15 can drive a motor program in the isolated cerebral ganglion}

At its resting potential $(-44.4 \pm 3.8 \mathrm{mV}, n=15) \mathrm{C} 15$ usually received no large spontaneous synaptic inputs and was silent (e.g., Figs. $3 A, 4,8 C$ ) or weakly tonically active (e.g., Figs. $3 B, 8 A, B$ ). Some low-amplitude spontaneous input was present, because the resting membrane potential showed less short-term variations in the presence of $3 \times \mathrm{Ca}^{2+}, 3 \times \mathrm{Mg}^{2+}$, which presumably reduced presynaptic spiking activity (Fig. 2D,E). When $\mathrm{C} 15$ was fired at frequencies above 11-12 Hz, rhythmic synaptic activity with a cycle period of between 9 and $50 \mathrm{sec}$ was recorded in a variety of E- and M-cluster neurons (Figs. 3, 4, 8). In 25 out of 30 preparations, C15 produced repeating cycles of the program. In the remaining cases, only a single cycle was initiated, despite continued high-frequency firing of C15. Fast PSPs observed in some neurons (see below) were not one-for-one with spikes in C15 and they were completely abolished by $3 \times \mathrm{Ca}^{2+}, 3 \times \mathrm{Mg}^{2+}$. This indicates that the responses detailed below were not monosynaptic from $\mathrm{C} 15$, but arose through a polysynaptic pathway, after the activation of a cerebral CPG. The rhythmic activity could be induced in the isolated cerebral ganglion and, because it incorporated firing of lip motoneurons (see below) at frequencies that produce lip movements, it can be defined as a cerebral motor program (CMP). The CMP stopped within $100 \mathrm{msec}$, after cessathe of current injection in $\mathrm{C} 15$, even if this occurred in the middle reliably drive the CMP in preparations in which the cerebral ganglion had been isolated for up to $36 \mathrm{hr}$. C16 and C17 could investigated. 
A

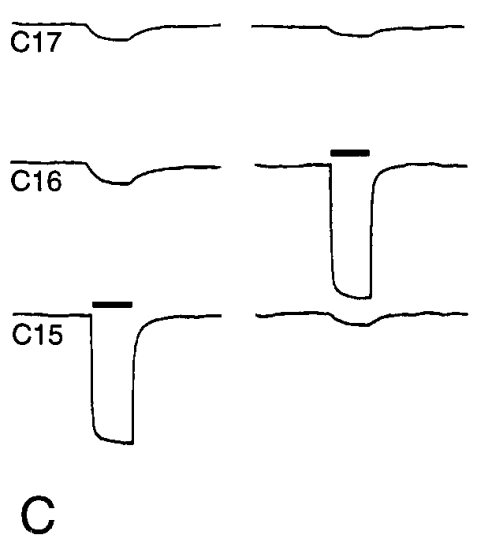

normal ASW

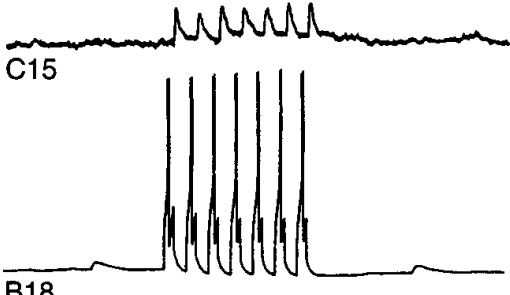

B18
D

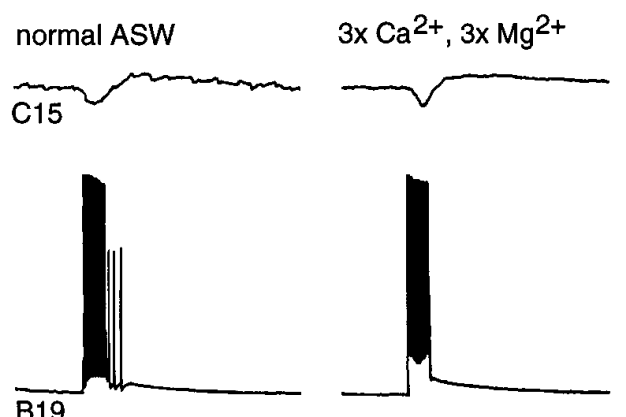

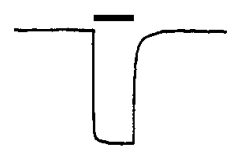

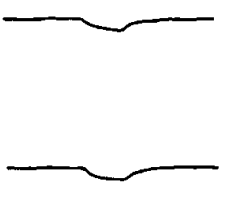

B

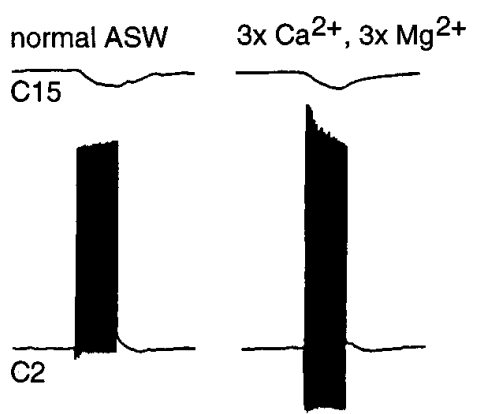

$3 \times \mathrm{Ca}^{2+}, 3 \times \mathrm{Mg}^{2+}$

Mum

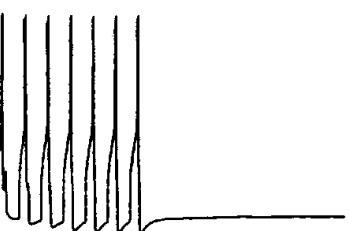

Figure 2. Synaptic connections onto C15. $A$, Lip motoneurons $C 15, C 16$, and $C 17$ were electrically coupled to each other. Negative current injected into any of the three cells (solid bars) produced a smaller hyperpolarization in the other two. $B$, Firing in the histaminergic mechanoafferent neuron $C 2$ produced slow inhibition in $C 15$. $C-E$, Spikes in BCIs B18, $B 19$, and $B 24$ produced, respectively, fast one-for-one EPSPs, an I/EPSP, and an IPSP in $C 15$. These responses all persisted in a solution containing high concentrations of divalent cations, which suppresses polysynaptic pathways. Scale bar: vertical $=40 \mathrm{mV}$ except $C 15$ in $C-E=15$ $\mathrm{mV}$; horizontal $=1 \mathrm{sec}$ for $C, 2 \mathrm{sec}$ for $A$, $10 \mathrm{sec}$ for $B, D$, and $E$.
To characterize more fully the CMP, we investigated the input received by a variety of identified cerebral neurons. Lip motoneurons $\mathrm{C} 11$ and $\mathrm{C} 12$ were active during the CMP (Fig. $3 A$ ). Firing in C11 was caused by barrages of EPSPs, whereas firing in $\mathrm{C} 12$ relied, in part at least, on postinhibitory rebound from a barrage of IPSPs, because the cell could also fire a burst of spikes at the end of a negative current pulse. Lip motoneurons C16 and C17 received large, fast EPSPs that drove high-frequency firing (Fig. $3 B$ ). Because of the electrical coupling of these neurons to $\mathrm{C} 15$, they were also tonically depolarized throughout the period of current injection in $\mathrm{C} 15$. C15 itself also received rhythmic input (open bar in Fig. 3B), which caused higher-frequency spiking (15-20 Hz) during those periods. In most records, the exact nature of this input was obscured by the current-induced spikes but, in cases where the CMP continued for a few tens of milliseconds after the current pulse, the input could be seen to consist of fast EPSPs, similar to those seen in $\mathrm{C} 16$ and $\mathrm{C} 17$.

In addition to motoneurons, we also recorded from a sample of previously identified sensory and modulatory cerebral neurons that participate in feeding motor programs (Chiel et al., 1986). The E-cluster neuron $\mathrm{C} 4$ can modulate contractions of extrinsic buccal mass muscles produced by cerebral motoneurons (Chiel et al., 1986). During the CMP, C4 received large EPSPs that drove it above threshold (Fig. 4A). $\mathrm{C} 5$, which is neighboring $\mathrm{C} 4$ but whose function is unclear, received relatively small EPSPs, onefor-one with those in $\mathrm{C} 4$, but these had little influence on its firing rate (Fig. 4A). The giant serotonergic metacerebral cell (MCC), which exerts modulatory influences both centrally and peripherally during feeding (Weiss et al., 1978; Rosen et al., 1989), received rhythmic weak, slow excitation but did not fire (Fig. 4B). The histaminergic mechanosensory neuron $\mathrm{C} 2$, which has a powerful influence over many neurons involved in the cerebral feeding motor circuitry (Chiel et al., 1986), received fast IPSPs (Fig. 4B).

Pairwise recordings revealed that the fast PSPs described above occur synchronously in all neurons, suggesting a common source (see expanded time-base records in Figs. 3, 4A). Occasionally, there were also fast EPSPs in the MCC, associated with the peak of the slow depolarization, but these were not one-for-one with 


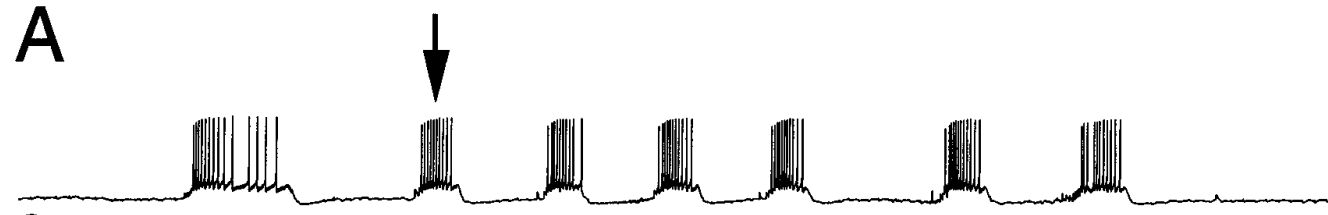

C11

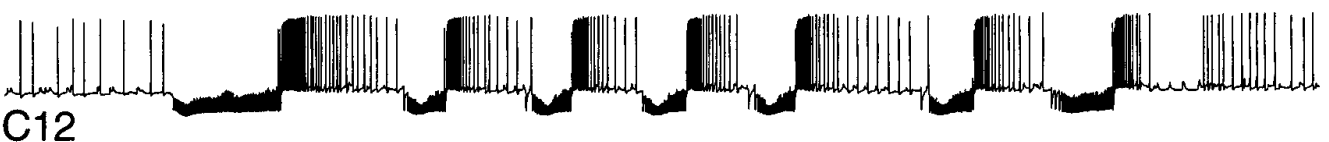

$\mathrm{C} 12$
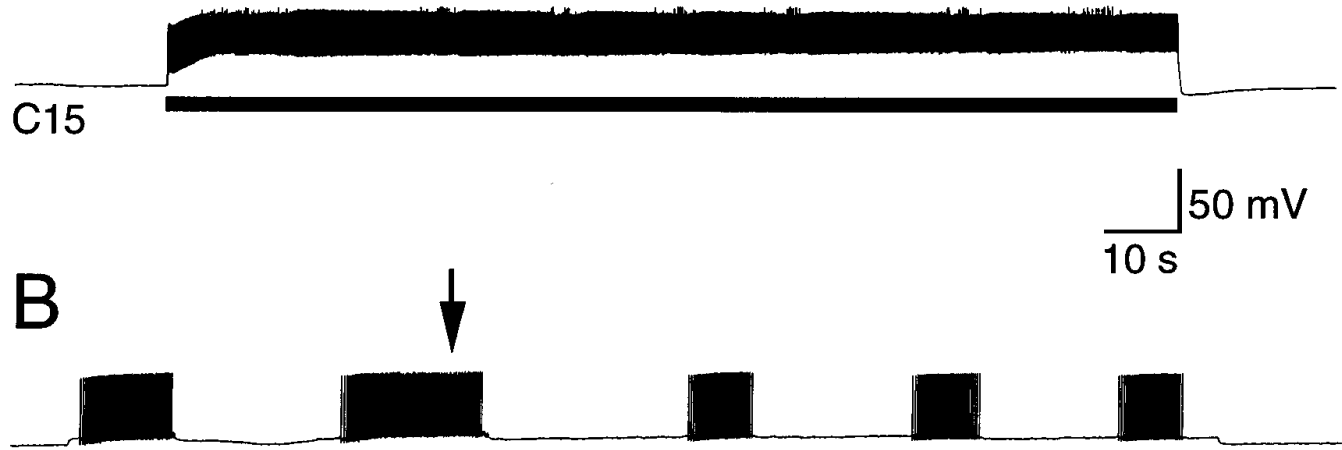

$\mathrm{C} 17$
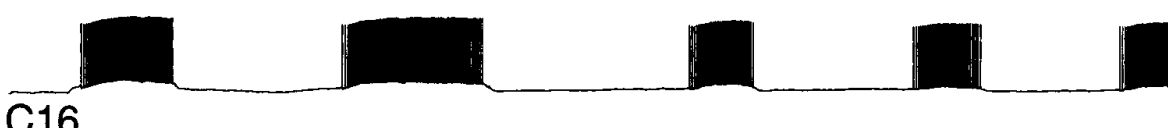

$\widehat{\mathrm{C} 16}$

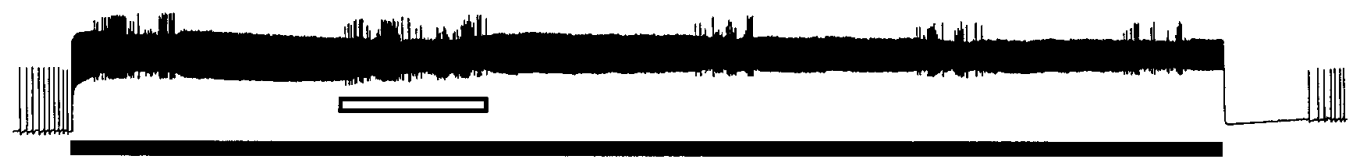

C15

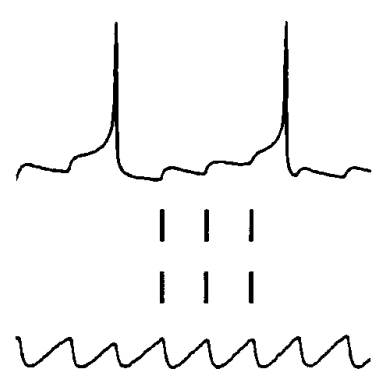

$50 \mathrm{mV}$
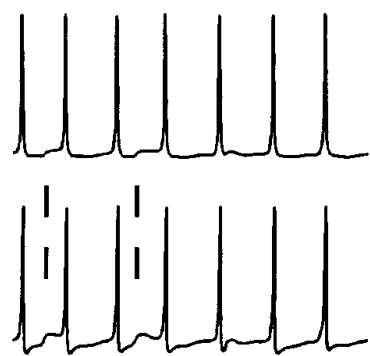

Figure 3. Input to cerebral lip motoneurons during the CMP in isolated cerebral ganglia. The CMP was driven by injecting constant depolarizing current into $C 15$ (solid bars). $A$, M-cluster lip motoneurons $C 11$ and $C 12$ were active in alternation. $C 11$ received EPSPs and $C 12$ IPSPs. B, E-cluster lip motoneurons $C 16$ and $C 17$ both received EPSPs during the CMP and fired at high frequency. $C 16$ and $C 17$ were electrically coupled to $C 15$ (and each other; see Fig. $2 A$ ) and so were also tonically depolarized throughout the current pulse. Arrows indicate points on the slow record from which the faster time-base records to the right were taken. These show that the PSPs in all neurons were one-for-one with PSPs in the others (examples joined by dashed lines), suggesting a common source. $C 15$ also received phasic excitatory input during the CMP (e.g., open bar in $B$ ) that was largely masked by the current-induced spiking but was represented by a rise in the firing frequency and uneven spike amplitudes.

the PSPs in the other cells (Fig. 4B). Only cells in the ipsilateral half of the ganglion appear to receive synaptic input during the CMP, because no rhythmic activity could be recorded contralaterally (Fig. 11A). There were only two discernible phases during the CMP, one being characterized by the presence of fast PSPs in the neurons described above and the other by firing in $\mathrm{C} 12$ and no observable input to any other neurons. The CMP, therefore, appears to be a less complex rhythm than the usually multiphase BMPs.

\section{Input to buccal motoneurons during the CMP}

In preparations with the buccal ganglion attached, a CMP that appeared identical to that produced in the isolated cerebral ganglion could still be driven by $\mathrm{C} 15$. Several identified cerebral-tobuccal interneurons (CBIs) fire during the CMP (see below) and are known to make synapses onto buccal motoneurons (Rosen et al., 1991b), so one might expect feedforward synaptic inputs from these neurons. Spiking of cerebral-to-buccal neurons was also monitored in isolated cerebral ganglia by an extracellular suction electrode placed on the $\mathrm{CBC}$. An increase in activity recorded in this electrode was always observed during the phase of the CMP in which fast PSPs occurred in $\mathrm{C} 2, \mathrm{C} 4, \mathrm{C} 5, \mathrm{C} 11, \mathrm{C} 12, \mathrm{C} 16$, and $\mathrm{C} 17$. This might be explained, in part, by the increase in spiking activity observed in CBI-2, CBI-3, and CBI-4 (described below). However, there was also a series of spikes in the $\mathrm{CBC}$ that were one-for-one with the fast PSPs in $\mathrm{C} 12$ (Fig. 5A). Firing in the identified CBIs occurred at a later phase than these spikes and was never one-for-one with the individual fast PSPs seen in other neurons (Figs. 8, 9B2,C2). This means there exists an as yet unidentified cerebral-to-buccal neuron that is responsible for this activity. Intracellular recordings from the buccal ganglion revealed that a number of neurons in the ventral motoneuron cluster received fast IPSPs during the CMP that were one-for-one with the fast PSPs in the cerebral ganglion (Fig. 5B). This suggests that the unidentified cerebral-to-buccal neuron makes synapses within the buccal ganglion, providing another source of input to buccal neurons, in addition to those arising via identified CBIs. 


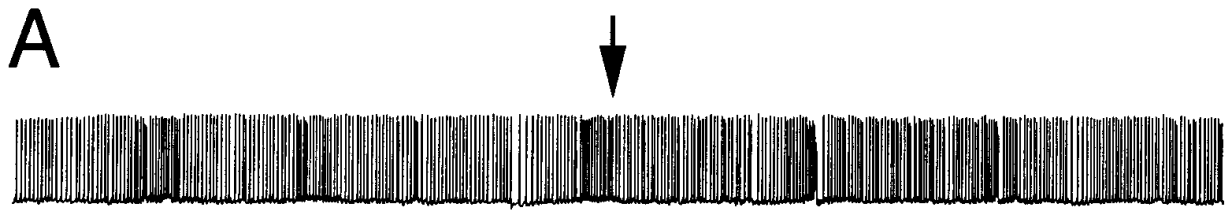

C5
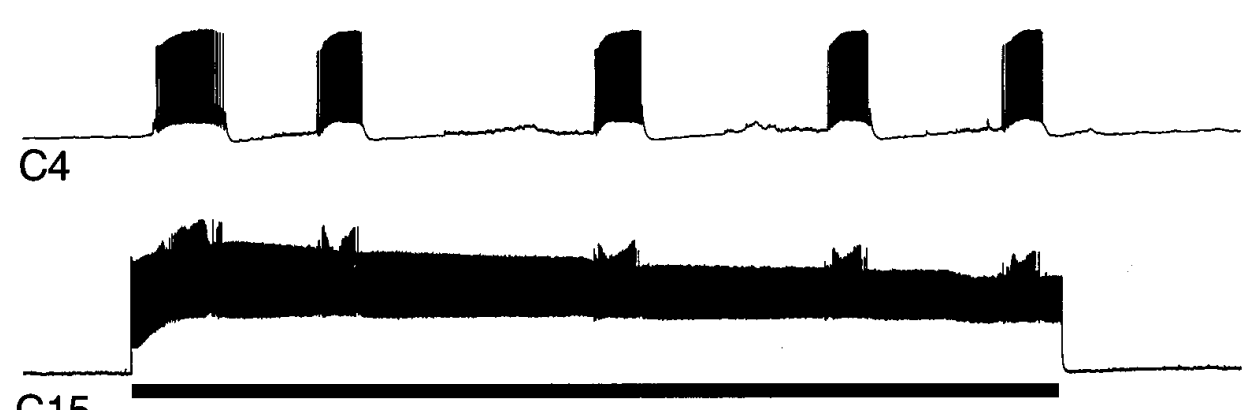

C15
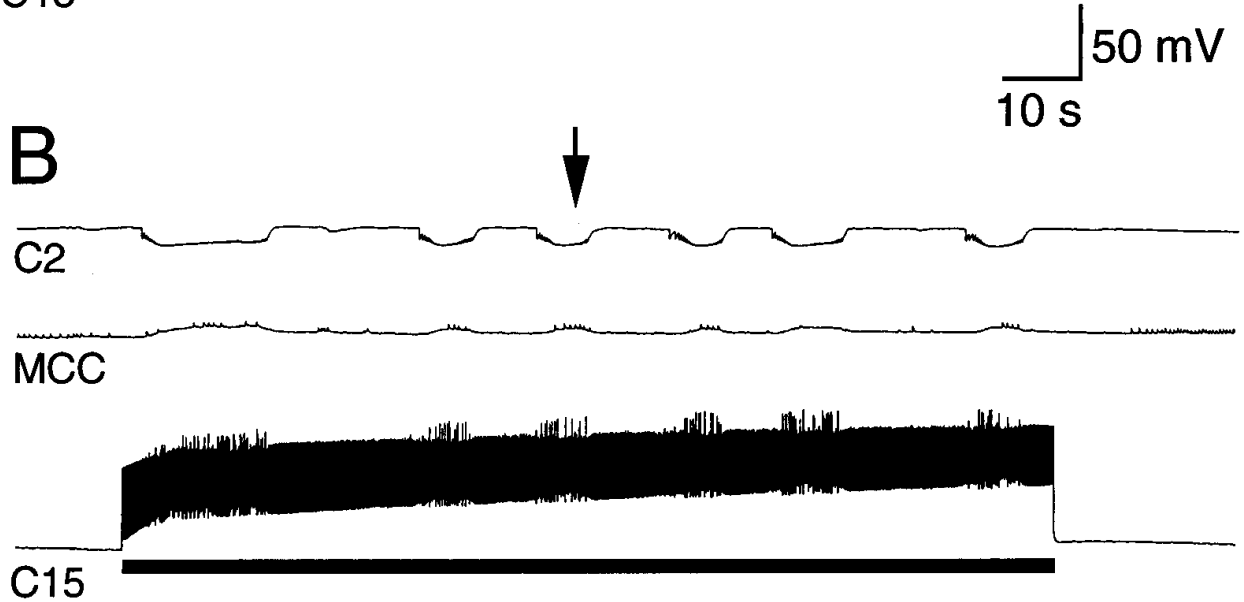
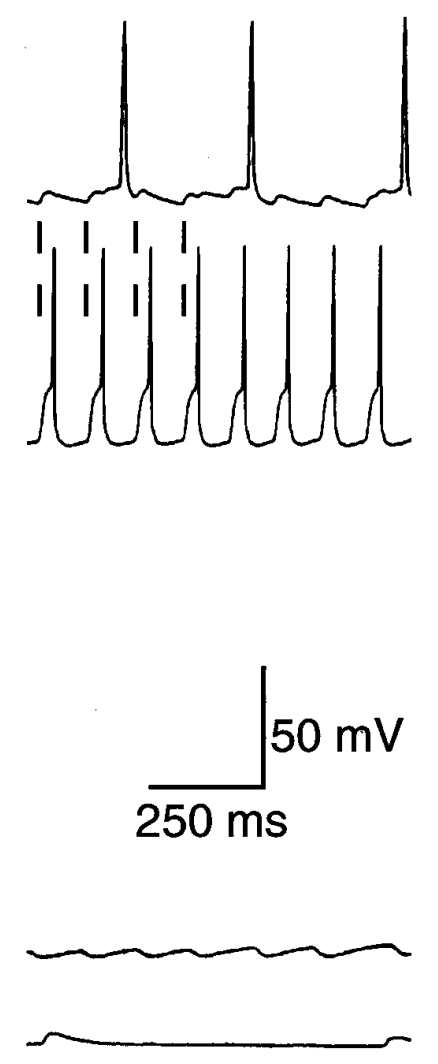

Figure 4. Input to modulatory and sensory neurons during the C15-induced CMP in isolated cerebral ganglia. $A$, Modulatory neuron $C 4$ received large EPSPs that drove it above threshold during the CMP, whereas its neighbor $C 5$ received much smaller EPSPs that were one-for-one with those in $C 4$ (see record to the right, showing input at an expanded time-base). These EPSPs had little effect on the firing rate of $C 5$. $B$, The sensory mechanoafferent $C 2$ received fast IPSPs during the CMP. The $M C C$, which has both central and peripheral modulatory roles, was slowly depolarized. There were also a few faster potentials in the MCC associated with the peak of some of the cyclical depolarizations, which were not one-for-one with the PSPs in other neurons (see record to the right). Arrows indicate points on the slow record from which the faster time-base records to the right were taken.

\section{The contribution of $\mathrm{C} 15$ to the CMP}

We have shown that $\mathrm{C} 15$ is sufficient to generate the CMP. To assess more fully the contribution of $\mathrm{C} 15$ to the operation of the cerebral CPG, we investigated if C15 firing was also necessary for the CMP (Kupfermann and Weiss, 1978). We therefore searched for other ways of driving the CMP to examine this possibility. In Pleurobranchaea, it has been shown that high-frequency stimulation of the $\mathrm{CBC}$ can drive a rhythm in the isolated cerebral ganglion (Davis et al., 1973; Cohan and Mpitsos, 1983), so we attempted the equivalent experiment in Aplysia. We found that stimulating the $\mathrm{CBC}$ at frequencies above $10 \mathrm{~Hz}$ resulted in rhythmic input to cerebral neurons (Fig. 6). Because lip motoneurons $\mathrm{C} 11, \mathrm{C} 15, \mathrm{C} 16$, and $\mathrm{C} 17$ were active during this rhythm, this can also be classified as a CMP. All of the recorded neurons had the same category of input during both $\mathrm{C} 15$ - and $\mathrm{CBC}$-driven CMPs. Thus, C4, C5, C11, C15, C16, and $\mathrm{C} 17$ all received fast EPSPs, whereas $\mathrm{C} 2$ and $\mathrm{C} 12$ received fast IPSPs. The cycle period of the CBC-driven CMP was 20-60 sec, which overlaps the values for the C15-driven rhythm. As with the C15-driven CMP, the source of these PSPs appeared to be an unidentified cerebral-to- buccal neuron, because the PSPs followed CBC stimulation with a fixed latency, regardless of stimulation intensity or frequency (Fig. $7 A$ ). Identified CBIs were ruled out as candidates because either no antidromic spikes were observed at the stimulus intensity used (CBI-2 and CBI-4) or blocking these spikes by hyperpolarization had no effect on the rhythm (CBI-1 and CBI-3). Because of these similarities, the CMPs driven by $\mathrm{C} 15$ depolarization or $\mathrm{CBC}$ stimulation appear to be very closely related and are likely to be produced by the same $\mathrm{CPG}$. To test whether spiking in $\mathrm{C} 15$ is necessary for the occurrence of the CMP, we hyperpolarized it during the $\mathrm{CBC}$-driven CMP to a level at which it no longer spiked. Under these conditions, the CMP could still be driven, suggesting that spiking in $\mathrm{C} 15$ is not necessary for the rhythm $(n=$ 6; Fig. 6B).

Using CBC stimulation, we were also able to investigate the nature of the possible connection between $\mathrm{C} 15$ and the unidentified cerebral-to-buccal neuron. For these experiments, the CBC was stimulated at low frequency $(1-2 \mathrm{~Hz}$, insufficient to induce the CMP) at an intensity just suprathreshold for the PSPs in C15, C12, etc. Under these conditions, hyperpolarizing C15 increased the 


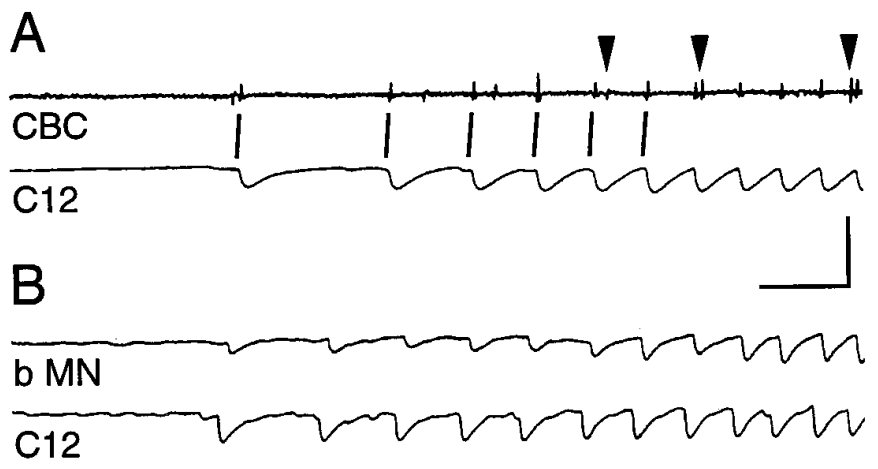

Figure 5. Feedback to the buccal ganglion during the CMP. A, A timeexpanded record of the start of one cycle of a CMP. A series of extracellularly recorded spikes in the $C B C$ were one-for-one with the IPSPs in $C 12$ (examples joined by lines). Because these were not attributable to spiking in any of the identified CBIs (see Results), these are the result of activity in an as yet uncharacterized cerebral-to-buccal neuron. There was also a general increase in the frequency of spikes in the $C B C$ (examples at arrowheads), possibly because of spiking in CBI-2, 3, and 4 (Fig. 8). B, A time-expanded record of the start of one cycle of a CMP. Facilitating IPSPs in an unidentified neuron $(b M N)$ in the ventral motoneuron cluster of the buccal ganglion were one-for-one with IPSPs in C12, suggesting a common source, probably the unidentified cerebral-to-buccal neuron. In both cases, the CMP was driven by constant current injected into $\mathrm{C} 15$ (not shown). Scale bar $=40 \mathrm{mV}$ for $C 12,10 \mathrm{mV}$ for $b M N$, and $250 \mathrm{msec}$ for $A, 500 \mathrm{msec}$ for $B$.

amplitude of the EPSP in that cell, without affecting the amplitude of PSPs in other cells (Fig. 7B1). However, we found that by further hyperpolarizing C15, we were able to block the PSPs in $\mathrm{C} 15$ as well as the PSPs in other neurons (Fig. 7B2). This suggests that $\mathrm{C} 15$ is electrically coupled to the unidentified cerebral-tobuccal neuron so that the hyperpolarization in C15 is transferred electrotonically, sufficiently hyperpolarizing the terminals of the other neuron to prevent transmitter release. It should be noted that a chemical synapse from the cerebral-to-buccal neuron onto $\mathrm{C} 15$ also exists, producing the EPSPs during the CMP, and that a chemical synapse in the other direction cannot be ruled out yet. The electrical connection between $\mathrm{C} 15$ and the cerebral-to-buccal neuron means that although spiking in $\mathrm{C} 15$ is not necessary for the CMP, a certain level of membrane potential is necessary. Thus, if $\mathrm{C} 15$ is strongly hyperpolarized (to presumably nonphysiological levels), it blocks the PSPs that follow CBC stimulation and, therefore, no program can be generated.

\section{Connections between cerebral and buccal CPGs}

Cerebral motoneurons receive input during BMPs (Rosen et al., 1991b) (see Figs. 10,11), and we have shown above that buccal motoneurons receive input during the CMP (Fig. 5). We also wished to characterize possible interactions between the cerebral and buccal CPGs themselves. To this end, we investigated whether neurons that are important during the operation of one type of program (either the CMP or various BMPs) received input during the other program. In Aplysia, we found the following. (1) Four identified cerebral-to-buccal interneurons (CBIs) are important in the control of BMPs (Rosen et al., 1991b) (see Fig. 10). We therefore investigated input received by these neurons during the CMP. (2) C15 is important in the control of the CMP (because it is sufficient to drive it and receives excitatory input during the program). We therefore investigated input received by this neuron during BMPs.

\section{Inputs to CBIs during the CMP}

CBI-1 received weak, slow inhibition during the CMP (Fig. $8 A$ ), which was not studied further. CBI-2 and CBI-4 both received slow excitation, which was usually sufficient to produce spiking (Fig. $8 B, 9 B, C$ ). Because firing $\mathrm{C} 15$ produced this excitation via a polysynaptic pathway (because the CMP was blocked in high divalent solutions), a detailed examination of the excitation in CBI-2 and CBI-4 was not attempted. However, it was apparent that in both cases the amplitude of the excitation was decreased by hyperpolarization of the CBIs (see Fig. 9B,C). CBI-3 was also excited during the CMP (Fig. $8 C$ ). We found that CBI-3 was strongly electrically coupled to $\mathrm{C} 4$. Injecting either a positive or a negative current into either $\mathrm{CBI}-3$ or $\mathrm{C} 4$ altered the membrane potential of the other (Fig. $8 D$ ), the coupling ratio being $0.20-$ 0.25 . Spikes in one cell produced transient depolarizations of the other with zero latency. Bathing the ganglion in ASW with $10 \mathrm{~mm}$ $\mathrm{Co}^{2+}$ had no effect on these potentials, so there appears to be no chemical connection. The excitation of CBI-3 during the CMP could be effectively mimicked by positive current injection into $\mathrm{C} 4$ (Fig. $8 E$ ). This suggests that the excitation of CBI-3 during the CMP was largely attributable to electrotonic transfer of the chemical EPSPs and spiking in $\mathrm{C} 4$ described above. The input to all of the CBIs occurred during the same phase as the fast PSPs in the cerebral neurons described previously, although the firing in CBI-2 and CBI-4 was somewhat delayed and lasted longer, presumably because of the slow nature of the excitation.

In preparations with cerebral and buccal ganglia connected, the same inputs were observed for each of the CBIs during the CMP. Inducing the CMP by exciting $\mathrm{C} 15$ most often had little effect on buccal motor output, monitored via the extracellular recording electrode on bn2 (Figs. 9B,C, 14). However, in a significant number of preparations ( 5 of 23 ) single cycles of a BMP (defined as strong, multiphasic activity in bn2 and high-frequency firing in B4) were associated with the first cycle of the CMP (Fig. 9A). In only one case did consecutive cycles of the CMP produce repeating cycles of a BMP. Both CBI-2 and CBI-4 can be excited beyond threshold during the CMP. Because spiking in either CBI-2 or CBI-4 can drive BMPs (Rosen et al., 1991b) (see Fig. 10), this is a possible pathway for the generation of BMPs. This was tested by altering the membrane potential of CBI-2 and CBI-4 in preparations with the buccal ganglion attached. In the six preparations investigated in this manner, BMPs were not induced by $\mathrm{C} 15$ stimulation with all other neurons at their resting potentials. However, a full cycle of a BMP could be generated by slightly depolarizing CBI-2 or CBI-4, thus increasing the frequency at which they fired during the CMP (Fig. 9B,C). These results are not direct evidence that the CMP occasionally generates BMPs via excitation of CBI-2 and CBI-4 (or the combined action of both), but do show that this pathway is feasible.

\section{Input to C15 during BMPS}

In cerebral-buccal ganglia preparations, we also investigated the input to C15 during BMPs evoked by a variety of means. During the ingestive-like BMP driven by positive current injection into CBI-2 (cf. Rosen et al., 1991b), C15 received fast EPSPs that drove spiking at high frequencies (maximum frequency sustained over $1 \mathrm{sec}=24.5 \pm 1.3 \mathrm{~Hz}, n=6$; Figs. $10 A, 11 B, 12 A)$. $\mathrm{C} 15$ was also excited, although more weakly (maximum firing frequency, $14.3 \pm 2.3 \mathrm{~Hz}, n=5$ ) during egestive-like BMPs (Fig. 10B,C). These were driven by a brief burst of high-frequency $(10 \mathrm{~Hz})$ electrical shocks to the radula nerve or by constant stimulation of the esophageal nerve at $2-3 \mathrm{~Hz}$ (Susswein and Byrne, 1988). C15 

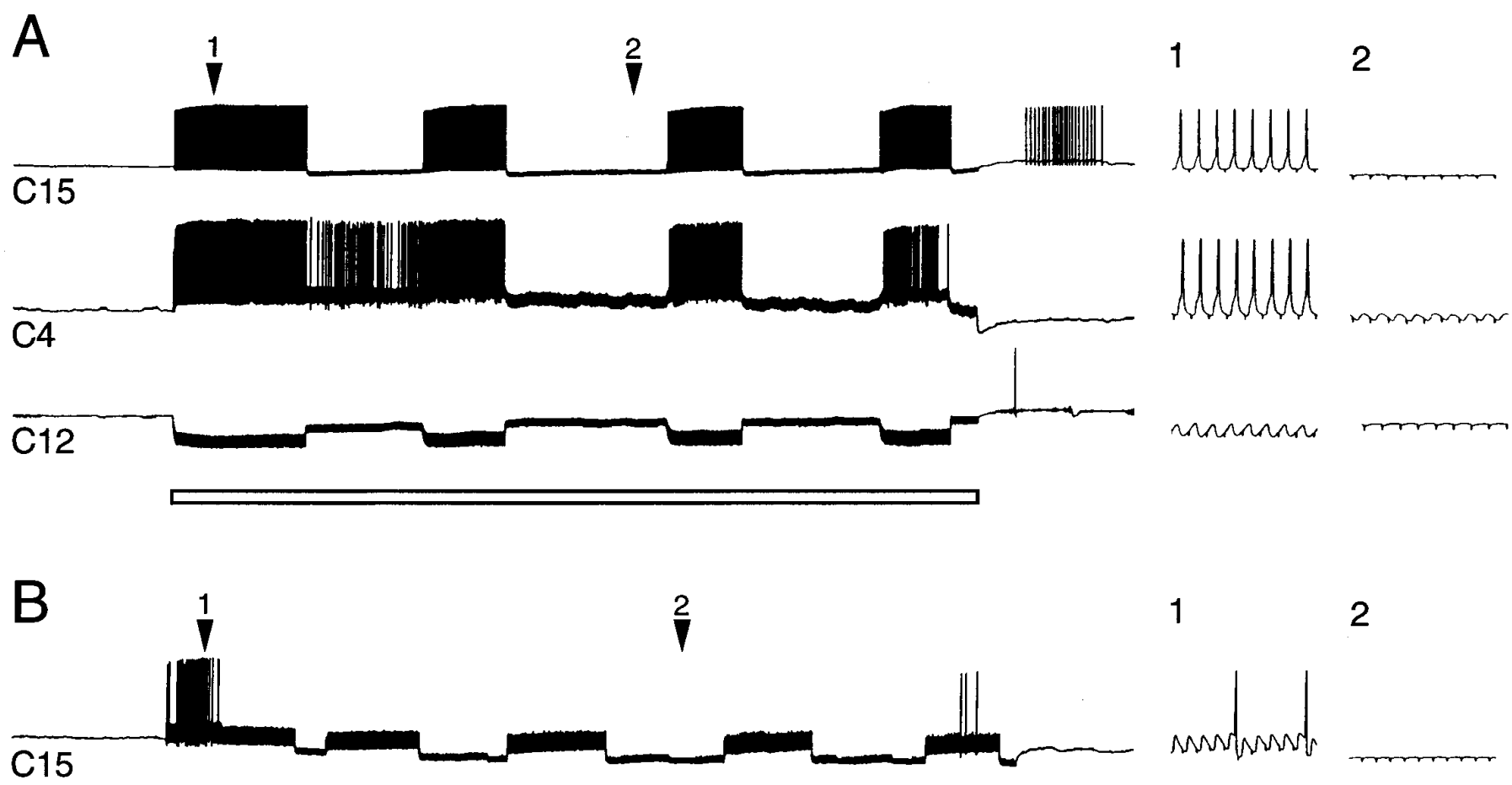

12
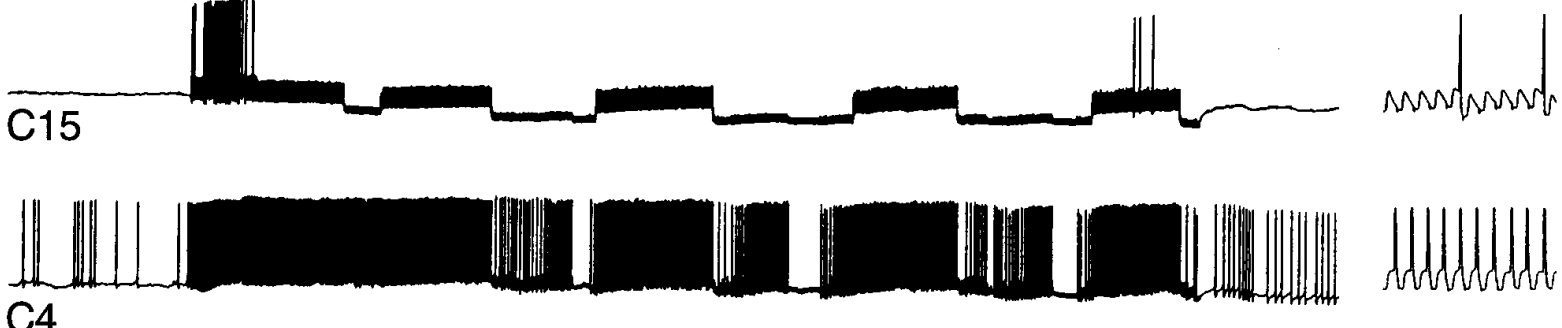

2

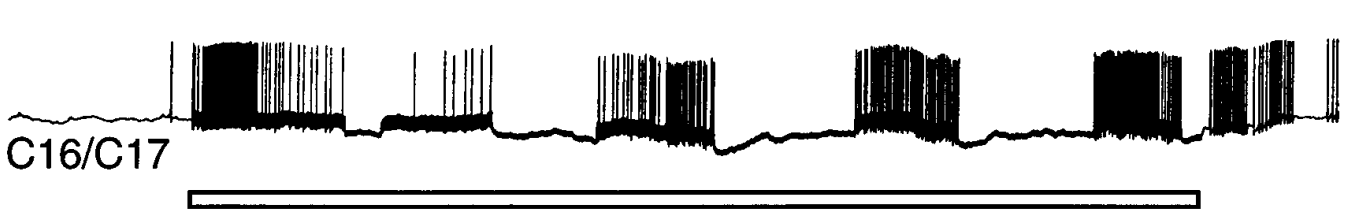

$25 \mathrm{~s}$
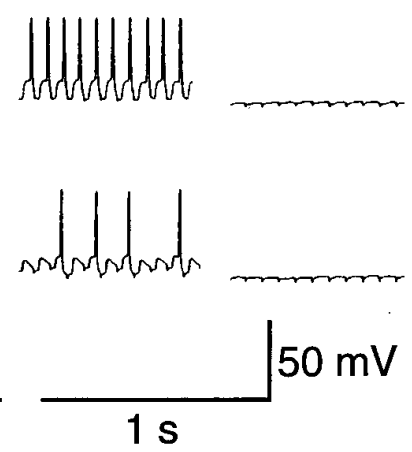

Figure 6. A CMP can be driven by high-frequency stimulation of the CBC. $A, C 15$, $C 4$, and $C 12$ all receive rhythmic input during CBC stimulation (open bar). The faster time-base records to the right show examples of the input to each neuron during the periods indicated by the numbered arrowheads on the slow record. The brief downward deflections represent CBC stimulus artifacts. $B$, In another preparation, stimulation of the CBC again resulted in rhythmic input, this time recorded in $C 15, C 4$, and $C 16 / C 17$. In this case, $C 15$ was hyperpolarized by $11 \mathrm{mV}$, which prevented any spiking during the middle three cycles of the CMP, showing that spiking in $C 15$ was not necessary for this CMP. Note that this CMP had a similar cycle period to the CMP driven by $C 15$ and that all of the neurons received similar inputs in both programs (compare with Figs. 3, 4).

was also excited during BMPs driven by both CBI-1 (maximum firing frequency of $\mathrm{C} 15=19.4 \pm 2.7 \mathrm{~Hz}, n=5$; Fig. 13A) and CBI-4 (maximum firing frequency $=16.0 \pm 1.5 \mathrm{~Hz}, n=6$; Figs. $10 D, 13 B)$. During BMPs initiated by any means, C15 was also occasionally inhibited between bursts (arrowheads, Figs. 10D, $11 B$ ). The CMP was strictly limited to one-half of the cerebral ganglion (Fig. 11 $A$ ), but the general timing of excitatory inputs to both right and left $\mathrm{C} 15 \mathrm{~s}$ was highly synchronized during BMPs (Fig. 11B), although individual EPSPs were not always one-for-one.

Although qualitatively similar (consisting of fast EPSPs), the input to C15 was not quantitatively the same for each variety of BMP. The firing rate of $\mathrm{C} 15$ was significantly higher $(p<0.01)$ during the BMP induced by CBI-2 (mean firing rate measured over the entire burst was $19.3 \pm 0.7 \mathrm{~Hz}, n=8)$ than that during CBI- 1 or CBI- 4 induced BMPs $(9.9 \pm 1.6 \mathrm{~Hz}, n=5$ and $9.5 \pm 0.9$ $\mathrm{Hz}, n=6$, respectively). Both of these firing rates were again significantly higher $(p<0.01)$ than during egestive-like BMPs $(4.3 \pm 1.0 \mathrm{~Hz}, n=6)$. Note that the maximum firing rates described above were also different between the various BMPs.

C15 was active during protraction for each type of BMP. Thus, during ingestive BMPs (Morton and Chiel, 1993b), such as that driven by CBI-2, buccal motoneurons $\mathrm{B} 8$ and $\mathrm{B} 4$ are active at the same time (Rosen et al., 1991b; Church and Lloyd, 1994). During this program, $\mathrm{C} 15$ was active before $\mathrm{B} 8 / \mathrm{B} 4$ (Fig. 10 $\mathrm{A}$ ), during protraction, and at the same time as "p-group" of neurons of Church and Lloyd (1994). During egestive-like motor programs, B8 and B4 are active out of phase (Morton and Chiel, 1993b; Church and Lloyd, 1994). In this program, C15 fires at the same time as B8 and stops before firing in B4 (Fig. 10B,C). This is also during protraction, but in this case at the same time as "c-group" neurons of Church and Lloyd (1994). C16 and C17 received similar input and were active at similar frequencies and with the same timing as $\mathrm{C} 15$. The timing of the activity in these lip 
A

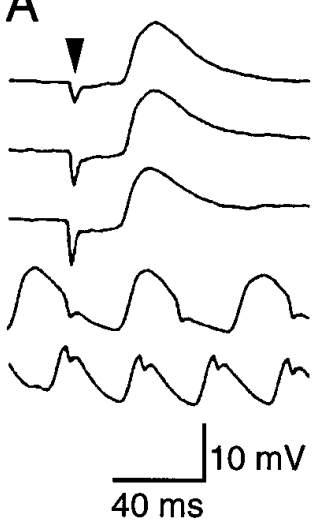

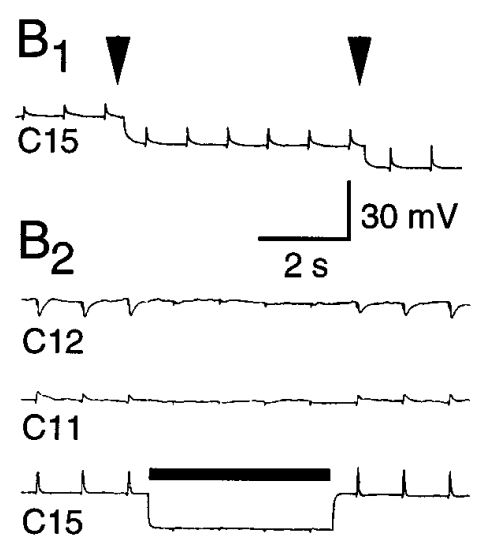

Figure 7. Properties of the PSPs evoked in cerebral neurons in response to CBC stimulation. $A$, The latency of the PSPs (in this case the EPSP in C15) was not affected by increasing the stimulation intensity (top three traces) or frequency (bottom two traces), indicating that the PSPs were probably attributable to a direct, monosynaptic connection from a cerebral-to-buccal neuron. The timing of $\mathrm{CBC}$ stimulation is indicated by the downward artifact at the arrowhead. B1, The EPSPs in C15 were classical chemical EPSPs, which increased in amplitude with increasing negative current (current injected at each arrowhead). B2, Hyperpolarizing C15 (during solid bar) beyond a threshold level completely abolished the EPSPs in C15, as well as the PSPs recorded in other cerebral neurons, in this case $C 11$ and $C 12$. This suggests that $C 15$ may be electrically coupled to the cerebral-to-buccal neuron responsible for the PSPs (see Results for details).

motoneurons relative to buccal motoneurons is consistent with the timing of jaw opening compared to radula movements during the two behaviors. During ingestion, the jaws are open while the radula is protracting and open, whereas during egestion the jaws are open while the radula is protracting and closed (Morton and Chiel, 1993a). It is possible that the BMP driven by CBI-4 underlies a swallowing behavior, so the second burst of spikes in B8, which coincides with inhibition of $\mathrm{C} 15$, during this motor program (Fig. 10D) may occur during hyper-retraction of the radula.

\section{The interactions between the cerebral and buccal CPGs change during different feeding-related motor programs}

Cerebral neurons receive input during BMPs partly as a result of direct, monosynaptic feedback from buccal-to-cerebral neurons, via axons in the CBC (Chiel et al., 1988; Teyke et al., 1993). However, during BMPs, $\mathrm{C} 15$ fired at frequencies that could drive the CMP. Because the cycle periods of BMPs were close to those for the CMP, C15 only fired at high frequencies long enough to drive part or all of a single CMP cycle for each BMP cycle. Nevertheless, this may be an important means of amplifying other synaptic inputs to some cerebral neurons during BMPs. We therefore investigated the effect of altering the membrane potential of $\mathrm{C} 15$ on input to cerebral neurons during BMPs. We generally used the large IPSPs observed in C12 during the CMP as a convenient monitor, but the results were also confirmed using the large EPSPs in C4.

For BMPs induced by CBI-2, large IPSPs were always observed in C12. These IPSPs were in phase with the excitation in C15, suggesting that the feedback loop through C15 to the cerebral CPG could contribute to their formation. If this were the case, then hyperpolarizing $\mathrm{C} 15$ might prevent the activation of the cerebral CPG, and so block some of these IPSPs. However, no reduction in the frequency of these IPSPs was observed even after quite extreme (and probably nonphysiological) hyperpolarization of $\mathrm{C} 15$ (Fig. 12).

In BMPs driven by both CBI-1 and CBI-4, large IPSPs in C12 were only sometimes observed (4 of 10 preparations), but when present were again in phase with the peak excitation in C15. In these types of BMP, however, the presence of IPSPs in C12 was critically dependent on the membrane potential of $\mathrm{C} 15$ (Fig. $13 A, B)$. If IPSPs were present, they could be blocked by hyperpolarizing C15 ( $n=4$; Fig. $13 A$ ). Conversely, if no IPSPs were present, they appeared if $\mathrm{C} 15$ was depolarized by $4-10 \mathrm{mV}$, below the threshold for driving the CMP in the quiescent state $(n=6$; Fig. 13B). This suggests that the IPSPs in C12 during these BMPs result from activity in the cerebral $\mathrm{CPG}$, driven at least partly by the phasic excitation in $\mathrm{C} 15$.

A third situation was observed for the egestive-like BMP driven by stimulation of the esophageal nerve. In this BMP, no large IPSPs were observed in $\mathrm{C} 12$ at any phase of the rhythm. Additionally, large IPSPs could not be induced by strong depolarization in $\mathrm{C} 15$ during the peak of its synaptic excitation (Fig. 14). During the egestive-like BMP, C12 received PSPs not correlated with the current pulse in $\mathrm{C} 15$, presumably attributable to direct input from BCIs. These PSPs caused a drop of $\sim 15 \%$ in the input resistance of $\mathrm{C} 12$ measured by constant hyperpolarizing current pulses. This relatively small change in the input resistance of $\mathrm{C} 12$ is unlikely to reduce the IPSPs during the CMP below detectable amplitude. For comparison, the decrease in input resistance in C12 caused by the IPSPs of the CMP itself was as much as $50 \%$. Even current levels well above the threshold for driving the CMP in the quiescent state could not drive the CMP during this BMP. Thus, not only does $\mathrm{C} 15$ fire at a lower frequency during the egestive-like BMP, but it is rendered incapable of driving the CMP.

\section{DISCUSSION}

\section{A separate cerebral CPG}

Our findings indicate that there is a CPG within the cerebral ganglion of Aplysia. In most other molluscan feeding systems, there is little evidence for separate cerebral CPGs, possibly because studies have concentrated on buccal motor circuitry. In Lymnaea, rhythmic synaptic inputs were recorded in some neurons in isolated cerebral ganglia (McCrohan, 1984), but the nature of this "oscillator" was not investigated further. In Pleurobranchaea, cerebral interneurons that drive buccal feeding rhythms [the phasic paracerebral (PCp) cells] also drive rhythmic motor output in isolated cerebral ganglia (Davis et al., 1984). The cerebral rhythm in Pleurobranchaea could only be obtained for about an hour after CBC transection. In Aplysia, however, the CMP could be activated in cerebral ganglia $36 \mathrm{hr}$ after CBC transection, showing that the cerebral CPG does not require short- or longterm feedback from the buccal ganglion to maintain its rhythmgenerating ability.

It should be noted that there are two separate cerebral CPGs, one in each hemiganglion, and each can be active independently. This feature may be important in producing asymmetrical lip movements during feeding behaviors if the exposure of seaweed to the lips is unilateral. Once the seaweed is positioned centrally and ingestion has begun, activity in the two cerebral CPGs will be synchronized because of simultaneous excitation of both C15s by the buccal CPG (Fig. 11), resulting in symmetrical lip movements.

The fast PSPs observed in cerebral neurons during the CMP do not represent monosynaptic connections from $\mathrm{C} 15$. The fact that 


\section{A}

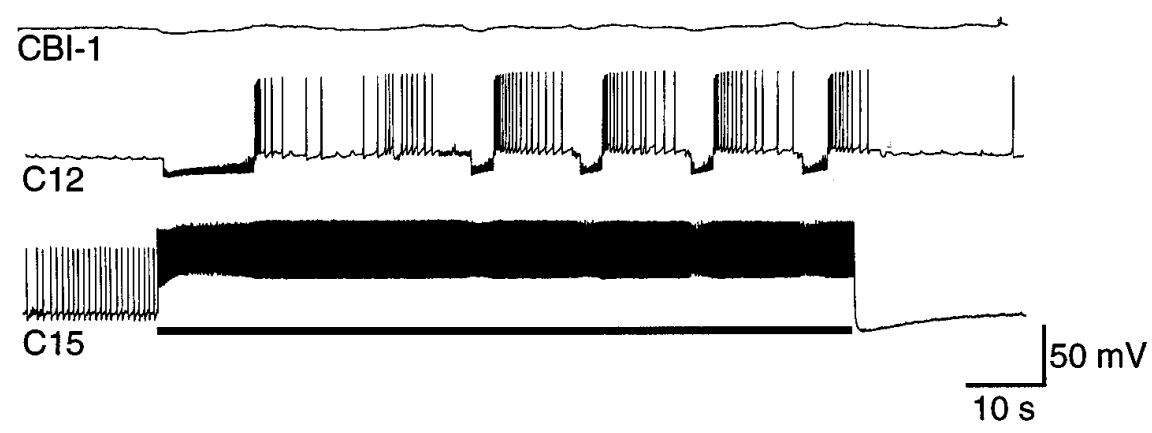

B

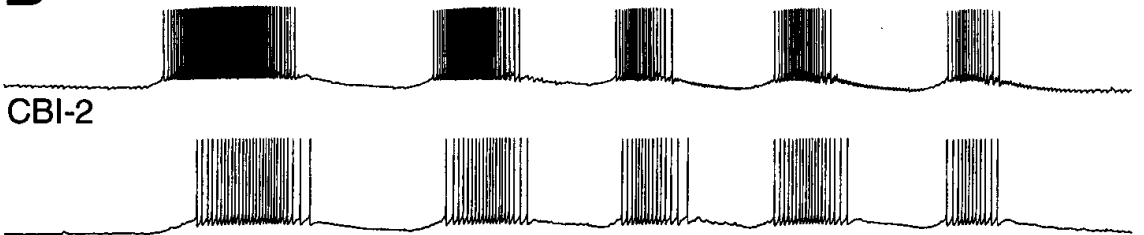

$\overline{\mathrm{CBI}-4}$

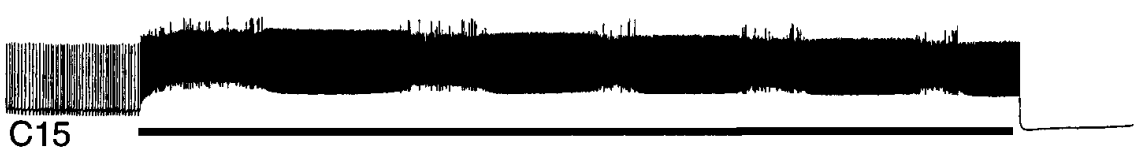

C

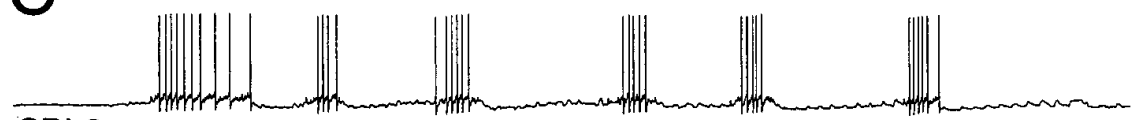

CBI-3
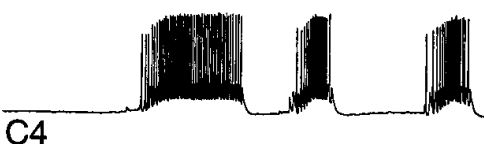

\section{e}

C4

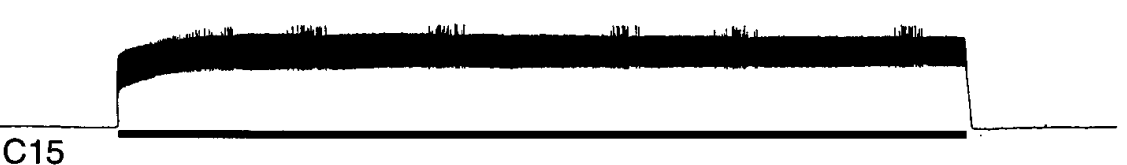

D
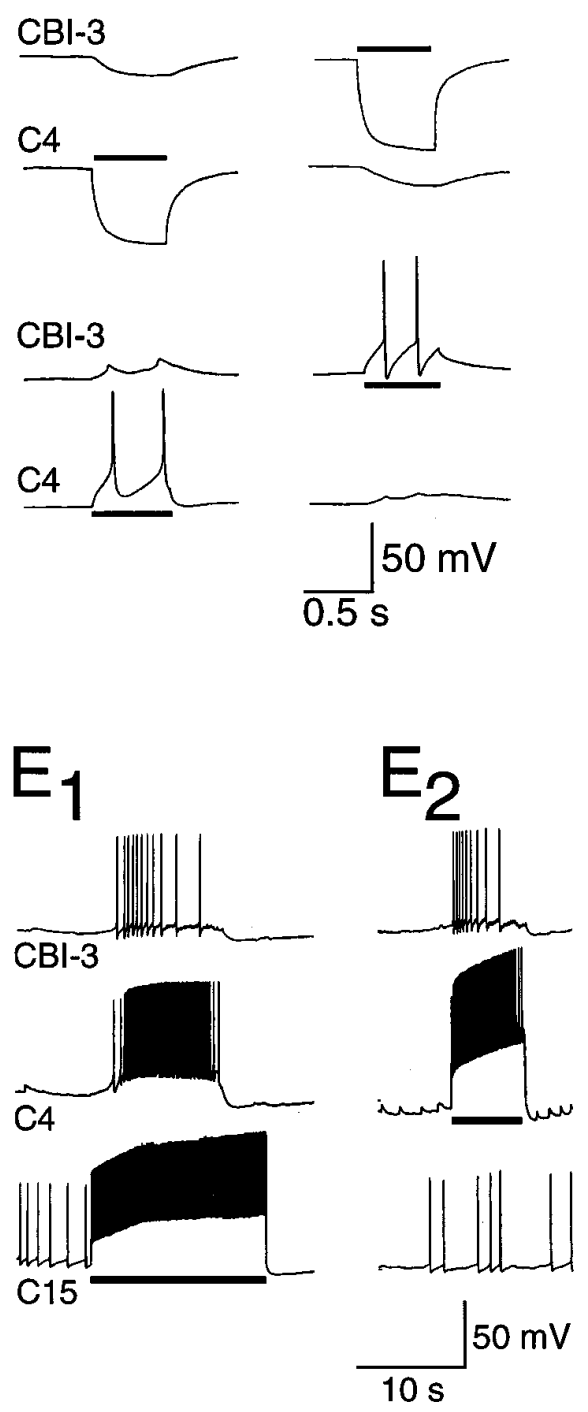

Figure 8. Input to CBIs during the CMP in the isolated cerebral ganglion. $A, C B I-1$ was weakly, slowly inhibited during the CMP, in phase with the fast IPSPs in $C 12 . B, C B I-2$ and $C B I-4$ received slow excitation during the CMP, which depolarized them above spiking threshold. $C$, $C B I-3$ was excited and spiked during the CMP, in phase with the IPSPs in C12 (not shown) and the EPSPs in C4. D, CBI-3 and C4 were electrically coupled, because hyperpolarizing or depolarizing current pulses in either neuron (solid bars) led to a smaller membrane potential change of the same polarity in the other. Action potentials in one neuron gave rise to brief depolarizing potentials in the other without a measurable delay. $E$, The excitation seen in CBI- 3 during a cycle of the CMP induced by firing of $C 15$ (E1) was effectively mimicked by a depolarizing current pulse into $C 4$ (E2), showing that electrotonic transmission of the chemically mediated excitation and spiking in $C 4$ is sufficient to explain the excitation recorded in $C B I-3$.

the PSPs are synchronous in all neurons suggests a common source, but this remains to be identified. It is likely that this common source is the unidentified cerebral-to-buccal neuron that produces (1) the spikes in the CBC that are one-for-one with PSPs in cerebral neurons during the C15-induced CMP, and (2) The constant-latency PSPs after $\mathrm{CBC}$ stimulation. The discovery of this neuron will be an important step in elucidating how the CMP is generated.
Because $\mathrm{C} 15$ is a lip motoneuron, driving the CMP via intracellular excitation of $\mathrm{C} 15$ caused strong tonic contraction of the inner lips that masked any superimposed rhythmic movements. However, during the CMP other cerebral motoneurons (such as $\mathrm{C} 16$ and $\mathrm{C} 17$ ) fire at frequencies that result in muscle contraction in the semi-intact preparation. Therefore, the cerebral CPG may be capable of producing rhythmic lip movements. Supporting evidence for such a role is provided by studies showing that lip 


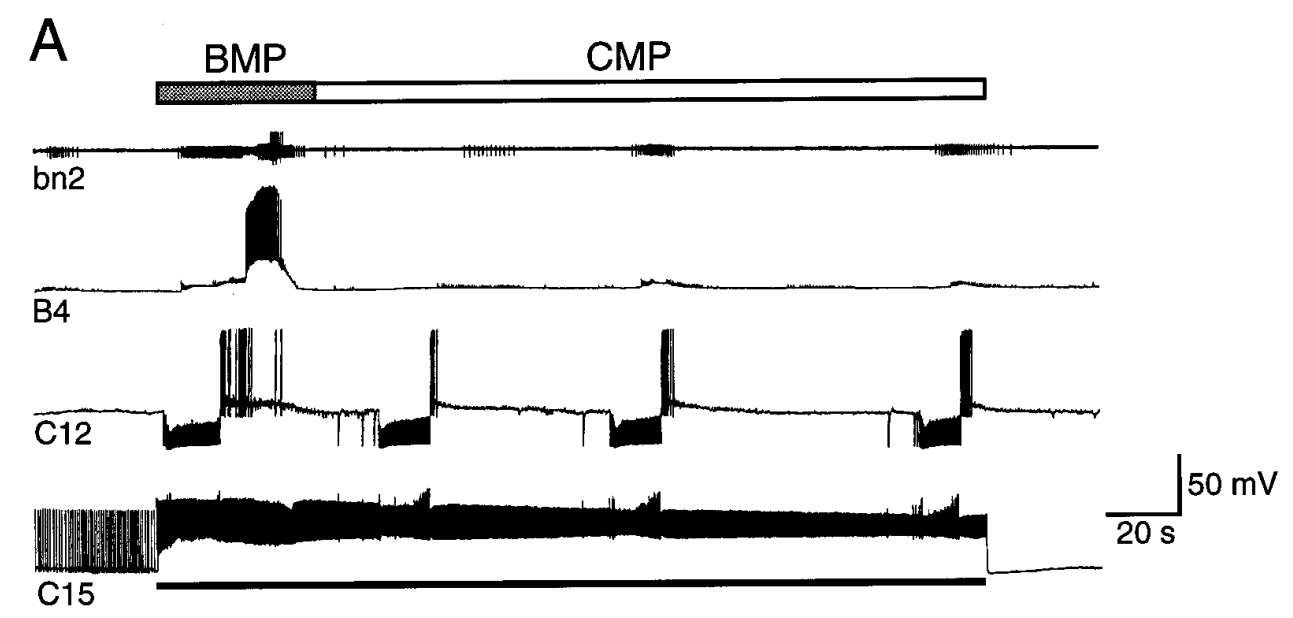

Figure 9. C15 initiated single cycles of a BMP, possibly via excitation of CBI-2 and CBI-4. A, Constant current injected into $C 15$ (solid bar) resulted in a single cycle of a BMP (gray bar), consisting of strong multiphasic motor output in $b n 2$ and firing in buccal neuron $B 4$. This is followed by repeating cycles of a CMP (open bar) with input to $C 12$, but only weak output in $b n 2$ and no firing in $B 4 . B$, With $C B I-2$ hyperpolarized $(B 1)$ or at its resting potential (B2), a $10 \mathrm{sec}$ pulse of depolarizing current into C15 (solid bars) resulted in a single cycle of a CMP with slow excitation in $C B I-2$, fast IPSPs in $C 12$ and no output in $b n 2 . B 3$, When $C B I-2$ was depolarized by $4 \mathrm{mV}$, it fired at high frequency during the CMP and a single cycle of a BMP was evoked, represented by multiphasic output in bn2 and feedback to $C B I-2$, $C 12$, and $C 15$. $C$, With $C B I-4$ hyperpolarized $(C 1)$ or at its resting potential $(C 2)$, a $10 \mathrm{sec}$ pulse of depolarizing current into C15 (solid bars) resulted in a single cycle of a CMP with slow excitation in $C B I-4$, fast IPSPs in $C 12$, and weak activity in $b n 2$. C 3 , When $C B I-4$ was depolarized by $5 \mathrm{mV}$, it fired at high frequency during the CMP and a single cycle of a BMP was evoked, represented by multiphasic output in $b n 2$ and feedback to $C 12$ and C15. Membrane potential of $C B I-2$ and $C B I-4$ is indicated at the right side of each trace.
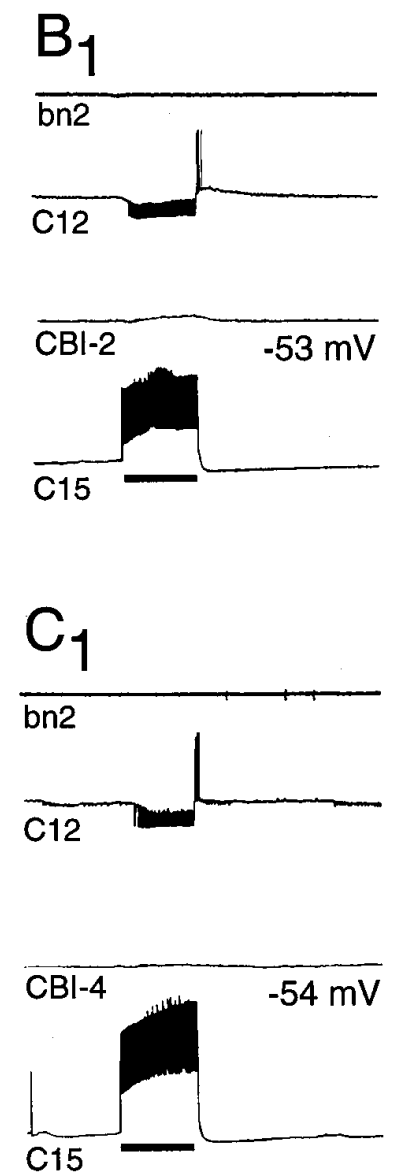
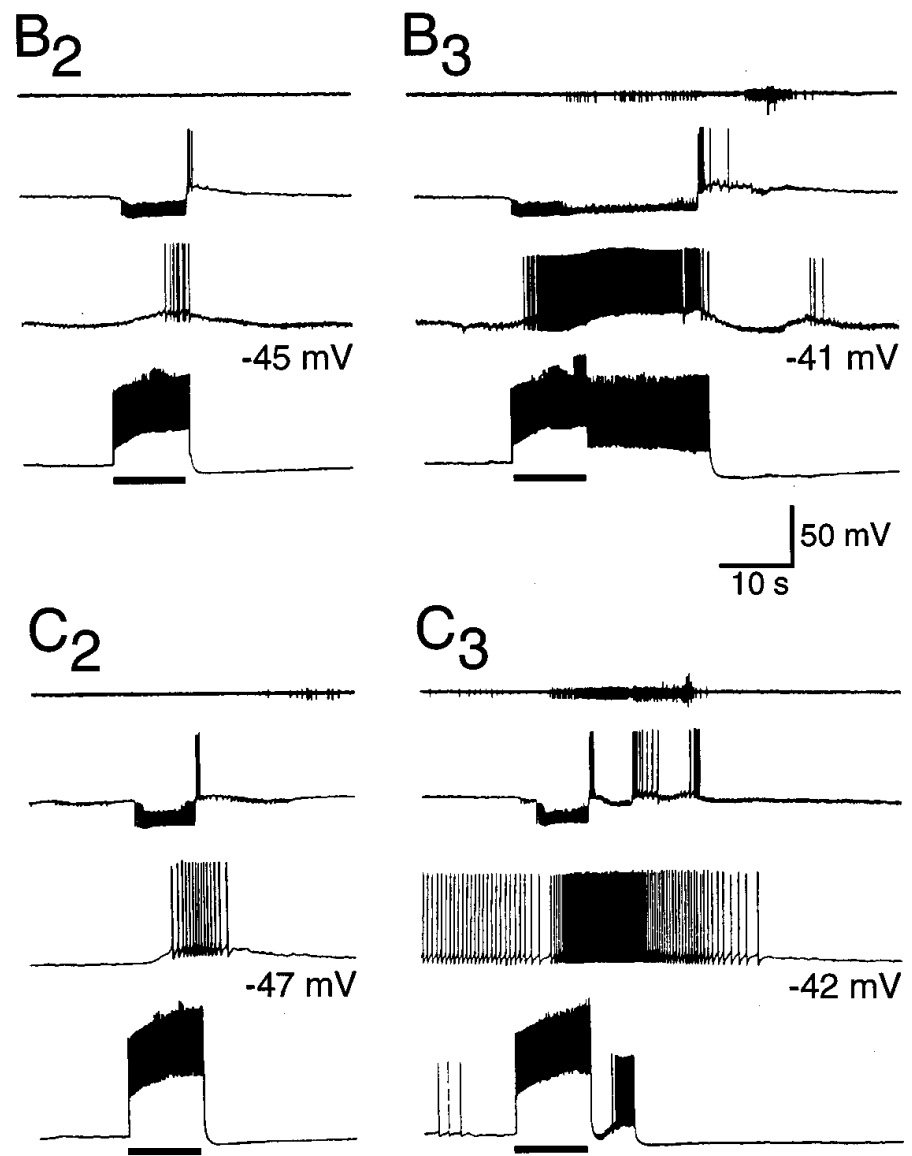

movements can occur after CBC transection (Kupfermann, 1974), or in the absence of ingestive movements of the buccal mass (during feeding: Hurwitz et al., 1996; during egg laying: Arch and Smock, 1977). Even if no behavioral correlate for the CMP is discovered, the changing interactions of cerebral and buccal CPGs are likely to play a role in shaping the coordination of lips and radula, as discussed below.

\section{The functional significance of $\mathbf{C 1 5}$}

$\mathrm{C} 15, \mathrm{C} 16$, and $\mathrm{C} 17$ cause contraction of the inner lips, resulting in jaw opening. These motoneurons are active at phases of ingestive and egestive BMPs when the jaws are opening and fire at a high

enough frequency during these BMPs to cause muscle contraction, so are likely to contribute to jaw opening during feeding behaviors. The higher firing rate of $\mathrm{C} 15$ during ingestive rather than egestive motor programs agrees with behavioral observations that the lips fully open during ingestion, but remain mostly closed during egestion (Morton and Chiel, 1993a).

C15 is a motoneuron capable of driving a CPG. There is evidence that motoneurons supply excitation to the vertebrate spinal locomotor pattern generator (Perrins and Roberts, 1995) and in several invertebrate systems motoneurons supply feedback to CPGs (Kristan and Calabrese, 1976; Heitler, 1978; Simmers 

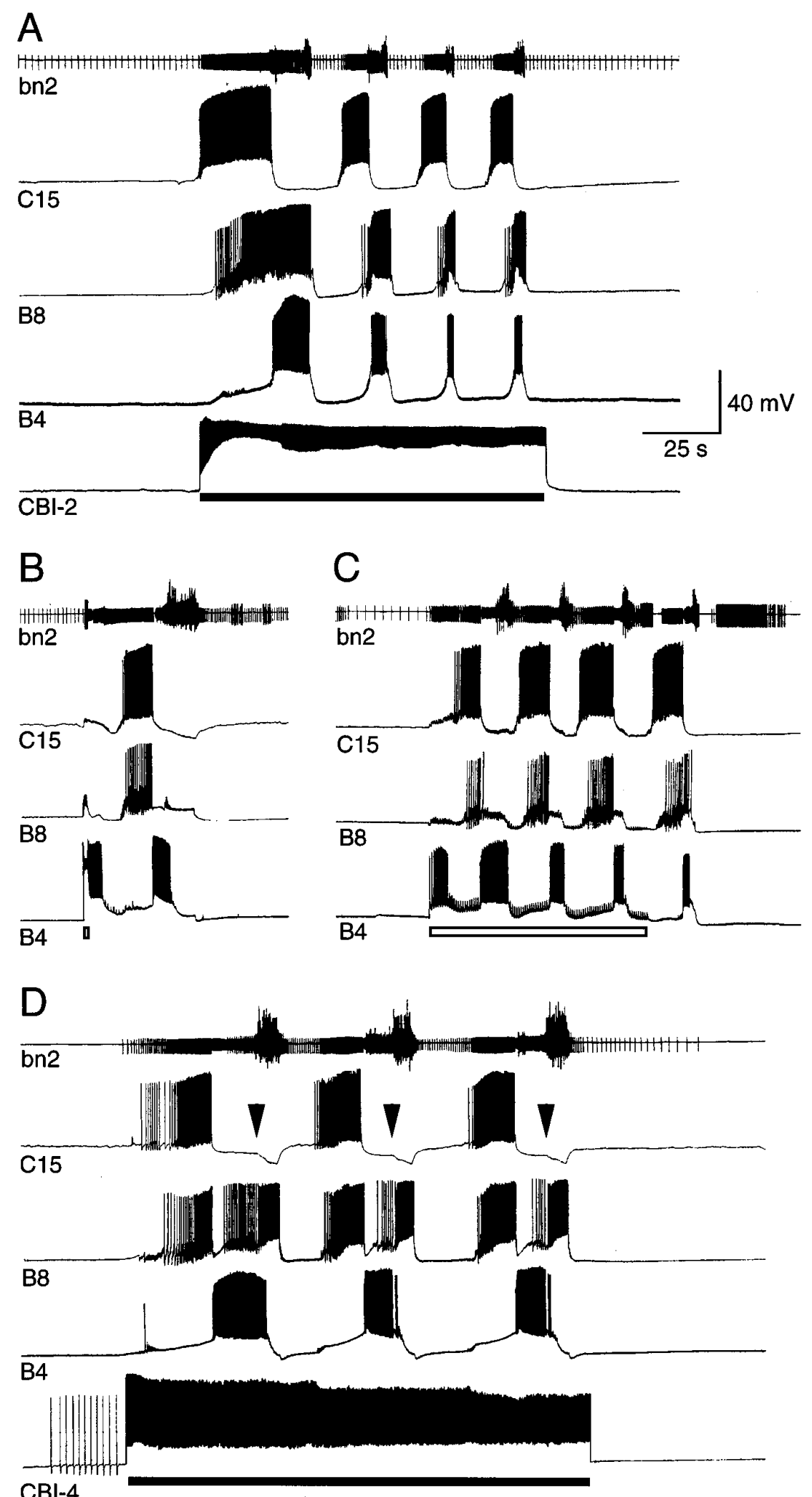

Figure 10. $\mathrm{C} 15$ received synaptic input during various BMPs. $A$, Constant depolarizing current injected into $C B I-2$ (solid bar) resulted in an ingestive-like BMP during which buccal neurons $B 8$ and $B 4$ fired together. During this program, $C 15$ was strongly excited just before the strongest activity in these neurons. $B$, During an egestive-like BMP induced by brief $10 \mathrm{~Hz}$ electrical stimulation of the radula nerve (during open bar), $B 8$ fires before $B 4$. In this program, $C 15$ was active at the same time as $B 8$ and before spiking in $B 4 . C$, During an egestive-like BMP induced by continuous 2.5 $\mathrm{Hz}$ stimulation of the esophageal nerve (open bar), C15 is again active with $B 8$, and before $B 4$. After the termination of nerve stimulation, there is a single cycle of a BMP that shows similar phase relations to the ingestive-like program shown in $A$. $D$, During a BMP induced by exciting $C B I-4$ (solid bar), $C 15$ was again active in phase with $B 8$ and before activity in $B 4$. In this program, there was a second burst of spikes in $B 8$ that coincided with inhibition in $C 15$ (arrowheads). For each BMP, the excitatory input to $C 15$ consisted of a barrage of fast EPSPs. Buccal motor output was also monitored from $b n 2$ in each case.

and Bush, 1983) or can drive a repeating rhythm (buccal CPGs of Tritonia: Willows, 1980; Aplysia: Hurwitz et al., 1994). C15 is also an important contact point between the cerebral and buccal CPGs, as discussed below.
Interactions between cerebral and buccal CPGs and their functional significance

It was previously thought that input to cerebral motoneurons was controlled by direct feedback from buccal circuitry (Fig. 15A). 


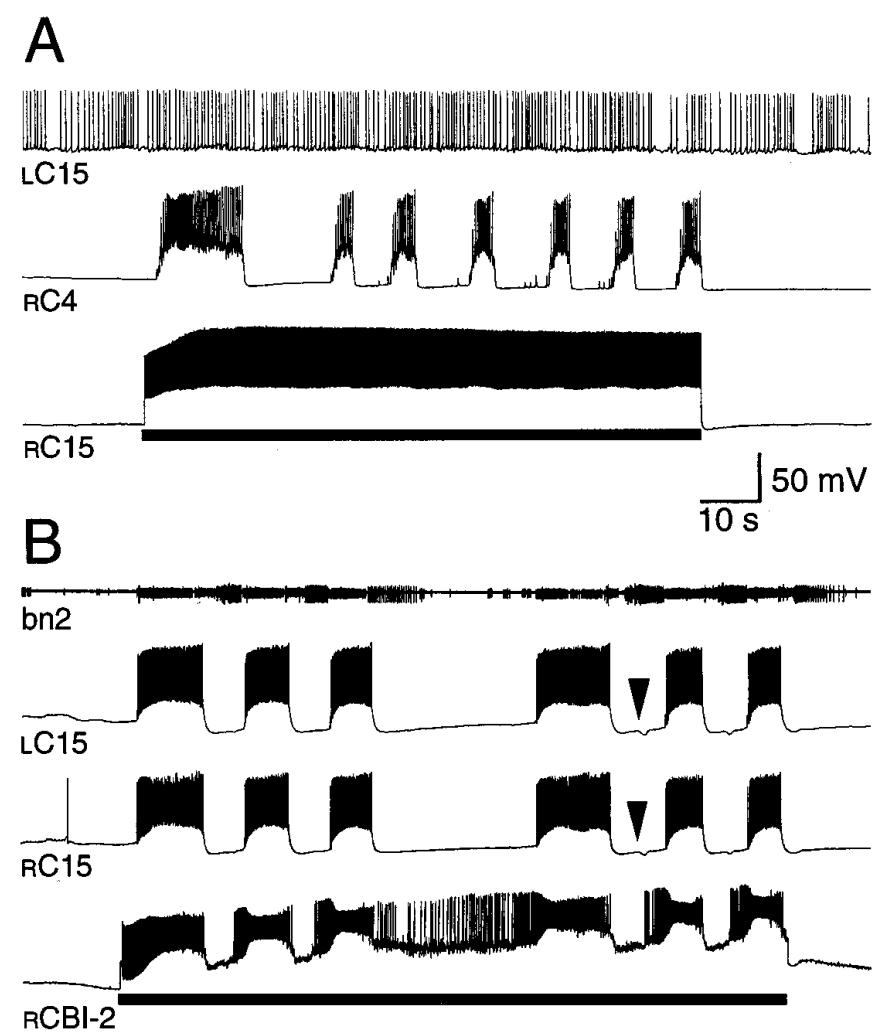

Figure 11. The CMP was restricted to the ipsilateral half of the cerebral ganglion, but contralateral $\mathrm{C} 15 \mathrm{~s}$ received synchronous input during BMPs. $A$, Constant depolarizing current injected into the right $\mathrm{C} 15(\mathrm{RC} 15)$ produced a CMP, demonstrated by input to the ipsilateral $\mathrm{C} 4(R C 4)$. However, no input was seen in contralateral neurons, in this case represented by the left $\mathrm{C} 15(L C 15)$. This suggests that there is a CPG in each cerebral hemiganglion that can operate independently. $B$, The $L C 15$ and $R C 15$ received synchronous excitatory input during a BMP driven by CBI-2. Thus, activity in the two hemiganglionic cerebral CPGs will be synchronized by buccal input during BMPs. Note that the occasional slow inhibitory input (arrowheads) was also synchronous.

Theoretically, the different coordination of lips and buccal mass seen during various feeding-related behaviors could be generated by a single buccal CPG if various motoneuron pools were decoupled during different behaviors. However, in this study we show the existence of a CPG in each cerebral hemiganglion that can also activate cerebral neurons and that interacts with buccal motor circuitry in various ways (Fig. 15B). Both buccal and cerebral CPGs have direct inputs to motoneurons in the other ganglion and, as previously thought, changes in the input from buccal circuitry to cerebral motoneurons will be vital in altering motor output during different behaviors. However, there are also other potential sites of modification.

The cerebral and buccal CPGs themselves are connected, because neurons that drive one program receive input during the other program. Thus, CBI-2 and CBI-4 (which drive BMPs) were excited during the CMP and, in turn, $\mathrm{C} 15$ (which drives the CMP) was excited during BMPs. It seems likely that the CPGs also interact in other ways. Rather than attempt to characterize all possible connections, we tried to ascertain whether such interactions alter during different motor programs. We show that for each variety of BMP (i.e., each BMP driven by a different means, such as intracellular stimulation of CBIs or esophageal nerve stimulation) the excitation in $\mathrm{C} 15$ changes both its amplitude and

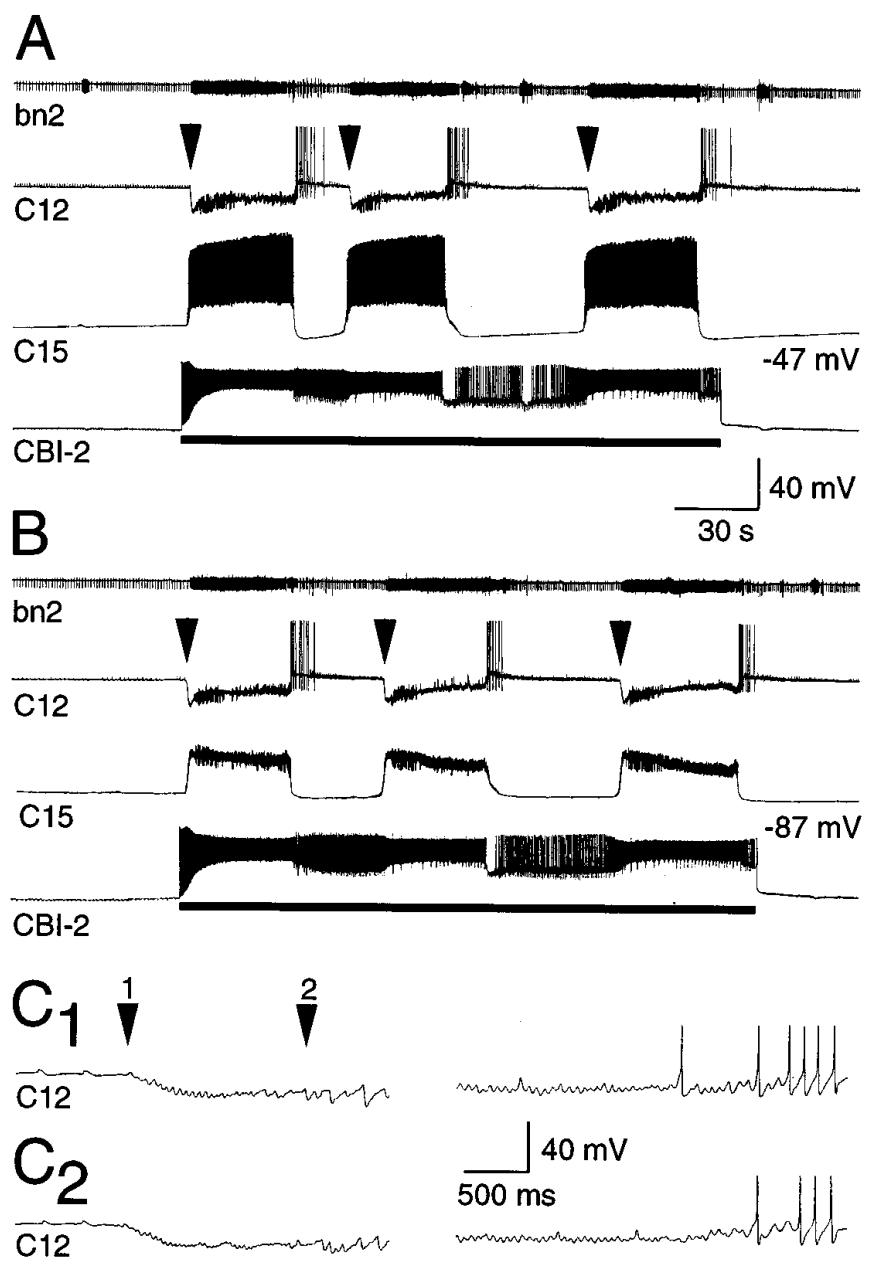

Figure 12. Input received by cerebral neurons during the CBI-2-driven $\mathrm{BMP}$ was not affected by altering the membrane potential of C15. A, With all neurons at their resting potentials, stimulation of $C B I-2$ (solid bar) resulted in a BMP with organized firing in $b n 2$ and input to cerebral neurons $C 12$ and $C 15$. The input to $C 12$ includes a barrage of fast IPSPs (at the arrowheads) that coincides with excitation in $C 15$. B, With $C 15$ hyperpolarized by $40 \mathrm{mV}$, it no longer fires during the $C B I$-2-driven BMP, although it still receives phasic excitation. Under these conditions, $C 12$ still receives IPSPs during the BMP. $C$ 1, Time-expanded records from $C 12$ showing the start and the end of the second barrage of IPSPs from $A$. Both small (arrow 1) and large IPSPs (arrow 2) can be seen. $C 2$, The equivalent record from $B$. The appearance and frequency of the IPSPs in $C 12$ are not affected when $\mathrm{C} 15$ is hyperpolarized.

its duration. Because $\mathrm{C} 15$ is a motoneuron, it could be argued that these changes reflect a need to change the extent and timing of jaw opening and have no functional relevance for interactions of buccal to cerebral CPG.

Evidence that interactions between the CPGs can be changed comes from experiments in which $\mathrm{C} 15$ alters the input received by other cerebral neurons (monitored using the large IPSPs in C12) during BMPs driven by some methods, but not others. We investigated BMPs driven by three means, as follows. (1) Depolarization of CBI-1 or CBI-4. Depolarizing C15 during these BMPs increased the number of IPSPs in C12, whereas hyperpolarizing $\mathrm{C} 15$ prevented them altogether. This suggests that the synaptic excitation received by $\mathrm{C} 15$ during these BMPs is sometimes sufficient to drive part of a cycle of activity in the cerebral CPG. Altering the excitation received by $\mathrm{C} 15$ by injecting positive or negative current either increases or sup- 

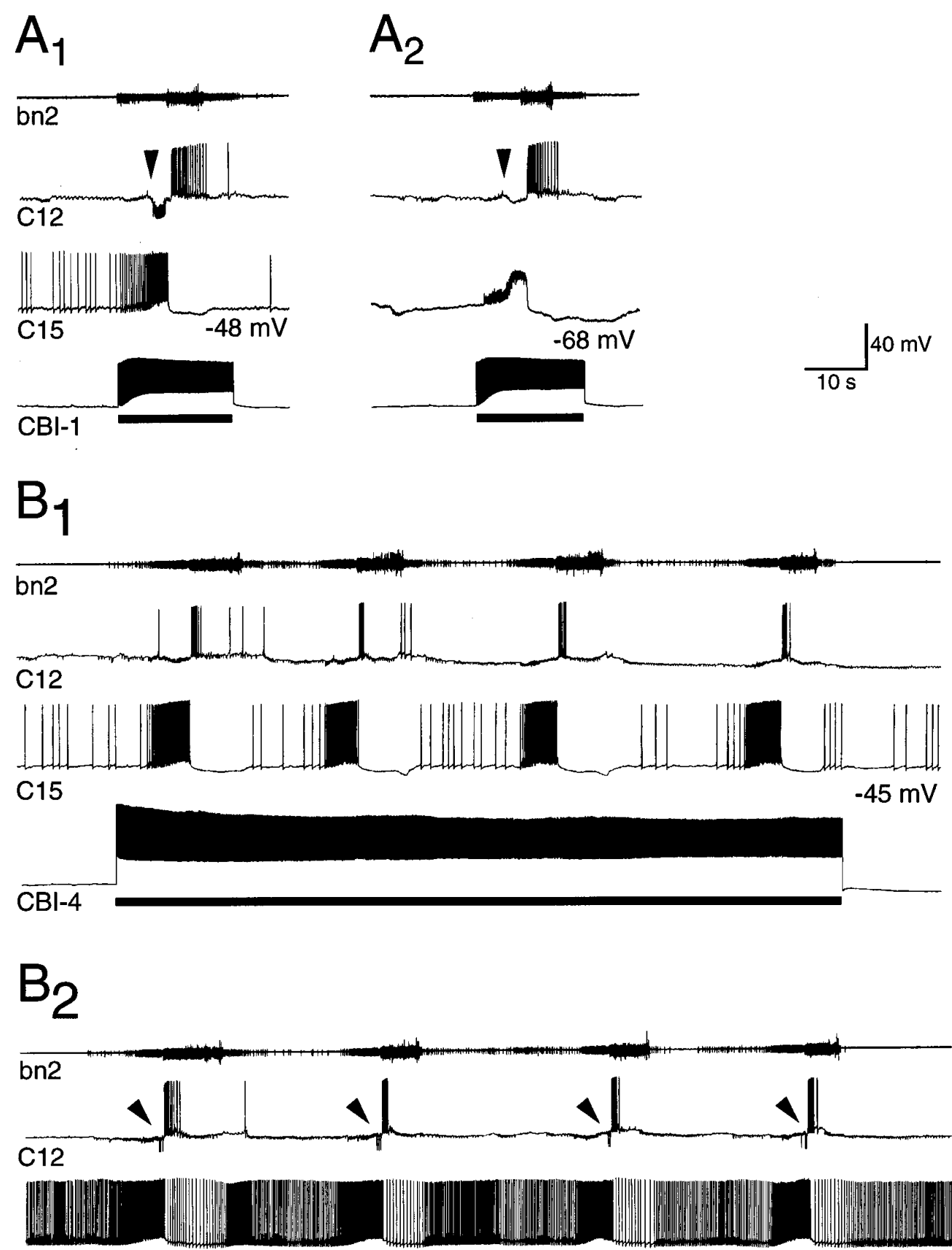

C15

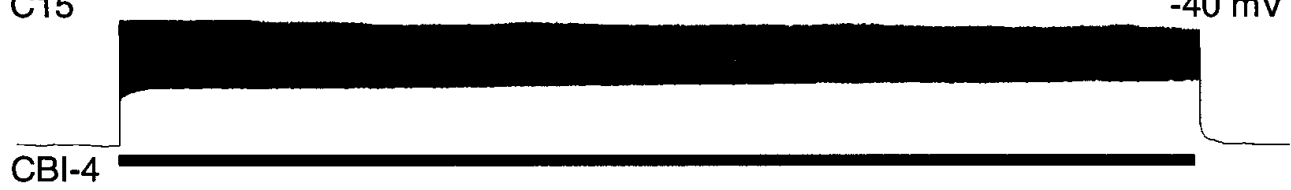

Figure 13. Input to cerebral neurons during CBI-1- and CBI-4-driven BMPs could be altered by changing the membrane potential of $\mathrm{C} 15$. A1, With all neurons at their resting potentials, stimulation of CBI-1 (solid bar) resulted in a single cycle of a BMP with organized firing in $b n 2$, and input to cerebral neurons $C 12$ and $C 15$. The input to $C 12$ included a barrage of fast IPSPs that was coincident with the peak depolarization of $C 15$ (arrowhead). A2, With $C 15$ hyperpolarized, the output of $b n 2$ remained the same but the fast IPSPs were no longer present in $\mathrm{C12}$. Note that even with C15 hyperpolarized $C 12$ still received some synaptic input during the BMPs. This presumably represents the direct feedback from the buccal $\mathrm{CPG}$, via BCIs. B1, With all neurons at their resting potentials, stimulation of $C B I-4$ (solid bar) resulted in repeating cycles of a BMP with organized firing in $b n 2$ and feedback to $C 12$ and $C 15$. No large IPSPs are present in C12. B2, $C 15$ is depolarized by $5 \mathrm{mV}$, so that it fired tonically at $5 \mathrm{~Hz}$, below the threshold for a CMP. During the BMP driven by $C B I-4$, the input to $C 12$ now includes a brief burst of 2-4 large IPSPs in each cycle (arrowheads), coincident with the maximum firing rate in $C 15$. When $C 15$ was depolarized by a further $3 \mathrm{mV}$, more IPSPs were observed in $C 12(6-25$ per cycle; not shown). presses this phasic activation of the cerebral CPG. The firing rate of $\mathrm{C} 15$ during naturally evoked behaviors could be altered by the various inhibitory and excitatory connections onto it, as shown in this study. For example, the mechanosensory neuron C2 inhibits C15 and is active during feeding (Chiel et al., 1986; Weiss et al., 1986). (2) Depolarization of CBI-2 (an ingestivelike BMP). In this program, large IPSPs were present in $\mathrm{C} 12$, regardless of the membrane potential of $\mathrm{C} 15$. There are three possible explanations for this. First, the IPSPs in C12 during this program may arise entirely from direct feedback from BCIs (connection $1 a$ in Fig. 15B). Second, the cerebral CPG may be so strongly excited by direct feedback from the buccal CPGs (connection 2a) that even extreme hyperpolarization of $\mathrm{C} 15$ cannot prevent its activation. Third, the connection from $\mathrm{C} 15$ to the cerebral CPG may be reduced in strength. (3) Stimulation of the esophageal nerve (an egestive-like BMP). Here, large IPSPs were not observed in $\mathrm{C} 12$ and could not generated by depolarization of $\mathrm{C} 15$ at levels suprathreshold for the CMP in the quiescent state. The inability of C15 to drive the CMP during this BMP might be attributable to decoupling at any of 


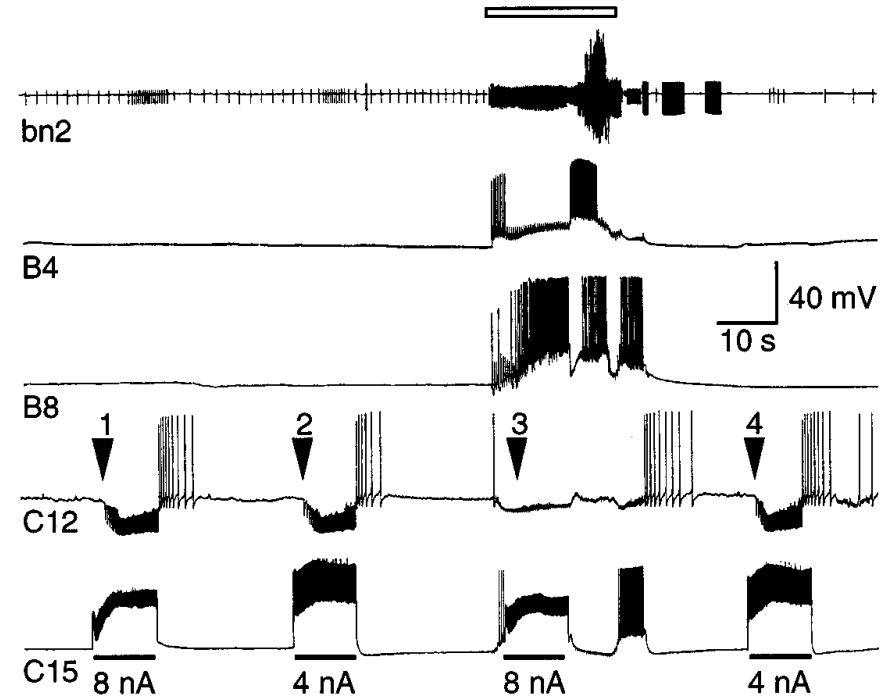

Figure 14. C15 could not drive a CMP during the egestive-like BMP induced by esophageal nerve stimulation. In the quiescent state, injecting $C 15$ with a $10 \mathrm{sec}$ pulse of either 8 or $4 \mathrm{nA}$ depolarizing current (filled bars) initiated a cycle of the CMP, shown by the barrage of IPSPs in C12 (arrowheads 1 and 2). A BMP was then driven by $2 \mathrm{~Hz}$ esophageal nerve stimulation (during open bar). $C 15$ was excited during this program, at the same time as $B 8$, and just before $B 4$ (compare Fig. 11 , and this synaptic drive is visible just before and after the current pulse). Depolarizing current ( $8 \mathrm{nA}$, previously suprathreshold for the CMP) injected into $C 15$ at the phase in which it received synaptic excitation could not drive a CMP, shown by the lack of large IPSPs in C12 (arrowhead 3). Soon after the end of the BMP, C15 could again drive the CMP (4 nA, arrowhead 4).

several sites. For example, the rhythm-generating ability of the cerebral CPG might be suppressed, the excitatory connection from $\mathrm{C} 15$ to the cerebral $\mathrm{CPG}$ might be reduced, or the synaptic outputs of the cerebral CPG might be inhibited.

How might activity in the cerebral CPGs contribute to specific aspects of feeding behavior? A possible role in generating asymmetrical lip movements was addressed earlier. Additionally, we can speculate how specific cerebral neurons may be influenced during protraction, the time when $\mathrm{C} 15$ receives excitation, thus possibly activating the CMP. For example, the mechanoafferent $\mathrm{C} 2$ is inhibited during the CMP and appears to be least active during protraction in the semi-intact preparation (Weiss et al., 1986). The CMP, therefore, might contribute to a relative enhancement of proprioceptive input from the lips and peri-oral zone during retraction, when seaweed is being ingested.

In conclusion, there are distinct buccal and cerebral CPGs, these CPGs interact at several points, and the interactions between these CPGs vary during different motor programs. Although the mechanisms underlying the changes in the interactions are not yet known, such variation does exist. Most obviously, C15 can drive the cerebral CPG during CBI- 1 and CBI-4 driven BMPs but cannot during egestive-like BMPs. These results lend support to two hypotheses: first, that there is often a separate CPG for each individual body part; second, that the interconnections between such CPGs alter during different motor programs. Future studies will need to determine whether such changes represent a mechanism for altering the coordination of separate body parts on a cycle-by-cycle basis.
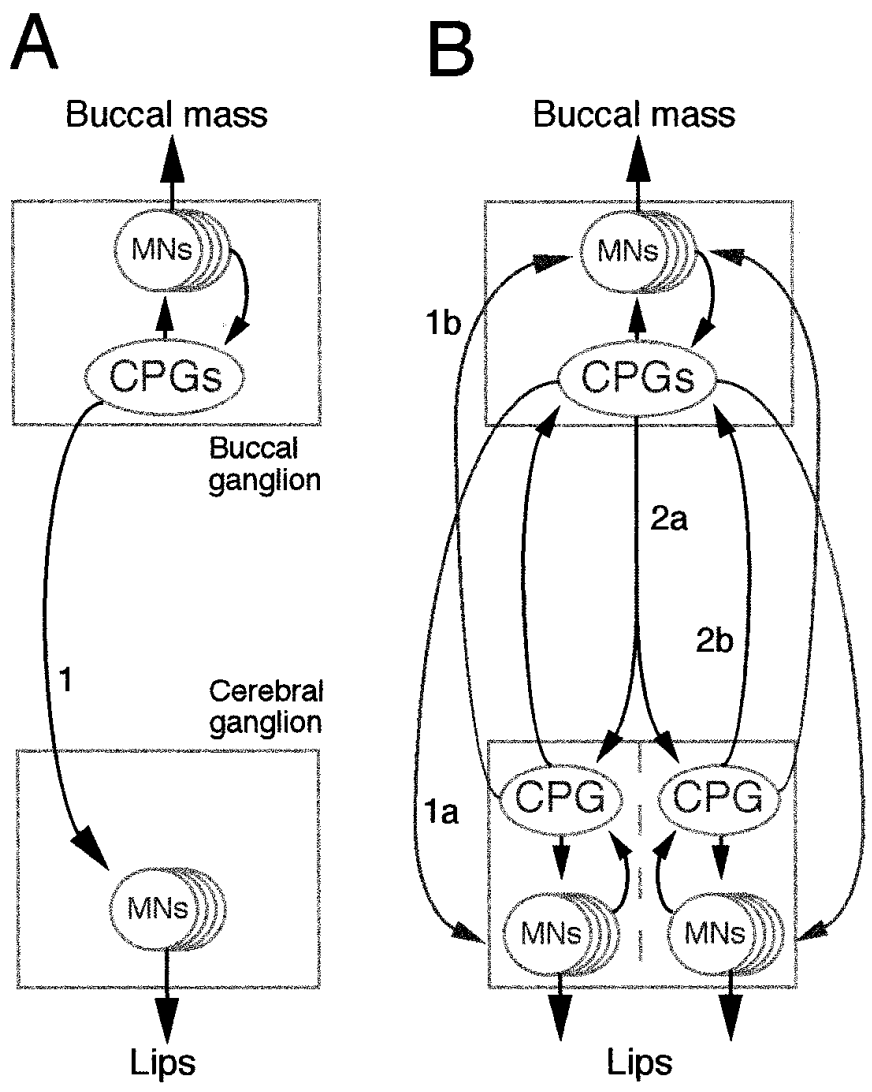

Figure 15. Summary diagram of the interactions between buccal and cerebral CPGs. $A$, Previously, it was thought that cerebral motoneurons that innervate the lips were controlled during feeding-related behaviors by direct feedback from one or more buccal CPGs (1). $B$, We now propose that there is a CPG in each cerebral hemiganglion that interact in variable ways with the buccal CPGs $(2 a, 2 b)$. Each CPG also makes direct connections onto motoneurons in the other ganglion $(1 a, 1 b)$. In both ganglia, arrows also indicate that there is feedback into the CPGs from some motoneurons (buccal: B31/32, Hurwitz et al., 1994; cerebral: C15, this study). The situation in $B$ allows greater flexibility than in $A$, because the output of the whole system can be altered by changing the strength or type of interaction at any of these points. Note that in both parts other known specific cerebral influences over buccal circuitry, such as the CBIs and MCC, are omitted for clarity.

\section{REFERENCES}

Arch S, Smock T (1977) Egg-laying behavior in Aplysia californica. Behav Biol 19:45-54.

Chiel HJ, Weiss KR, Kupferman I (1986) An identified histaminergic neuron modulates feeding motor circuitry in Aplysia. J Neurosci 6:2427-2450.

Chiel HJ, Kupferman I, Weiss KR (1988) An identified histaminergic neuron can modulate the outputs of buccal-cerebral interneurons in Aplysia via presynaptic inhibition. J Neurosci 8:49-63.

Church PJ, Lloyd PE (1994) Activity of multiple identified motor neurons recorded intracellularly during evoked feeding-like motor programs in Aplysia. J Neurophysiol 72:1794-1809.

Cohan CS, Mpitsos GJ (1983) The generation of rhythmic activity in a distributed motor system. J Exp Biol 102:25-42.

Cohen JL, Weiss KR, Kupferman I (1978) Motor control of buccal muscles in Aplysia. J Neurophysiol 41:157-180.

Davis WJ, Siegler MVS, Mpitsos GJ (1973) Distributed neuronal oscillators and efference copy in the feeding system of Pleurobranchaea. J Neurophysiol 36:258-274.

Davis WJ, Kovac MP, Croll RP, Matera EM (1984) Brain oscillators underlying rhythmic cerebral and buccal motor output in the mollusc, Pleurobranchaea californica. J Exp Biol 110:1-15.

Dickinson PS, Moulins M (1992) Interactions and combinations between different networks in the stomatogastric nervous system. In: Dynamic 
biological networks. (Harris-Warrick RM, Marder E, Selverston AI, Moulins M, eds), pp 139-160. Cambridge: MIT.

Friesen WO, Pearce RA (1993) Mechanisms of intersegmental coordination in leech locomotion. Semin Neurosci 5:41-47.

Grillner S, Wallen P (1985) Central pattern generators, with special reference to vertebrates. Annu Rev Neurosci 8:233-261.

Heitler WJ (1978) Coupled motoneurones are part of the crayfish swimmeret central oscillator. Nature 275:231-234.

Hurwitz I, Goldstein RS, Susswein AJ (1994) Compartmentalization of pattern-initiation and motor functions in the B31 and B32 neurons of the buccal ganglia of Aplysia californica. J Neurophysiol 71:1514-1527.

Hurwitz I, Neustadter D, Morton DW, Chiel HJ, Susswein AJ (1996) Activity patterns of the $\mathrm{B} 31 / \mathrm{B} 32$ pattern initiators innervating the I2 muscle of the buccal mass during normal feeding movements in Aplysia californica. J Neurophysiol 175:1309-1326.

Jahan-Parwar B, Fredman SM (1976) Cerebral ganglion of Aplysia: cellular organization and origin of nerves. Comp Biochem Physiol 54A:347-357.

Kirk MD (1989) Premotor neurons in the feeding system of Aplysia californica. J Neurobiol 20:497-512.

Kristan Jr WB, Calabrese RL (1976) Rhythmic swimming activity in neurons of the isolated nerve cord of the leech. J Exp Biol 65:643-668.

Kupfermann I (1974) Dissociation of the appetitive and consummatory phases of feeding behavior in Aplysia: a lesion study. Behav Biol 10:89-97.

Kupfermann I, Weiss KR (1978) The command neuron concept. Behav Brain Sci 1:3-39.

McCrohan CR (1984) Initiation of feeding motor output by an identified interneurone in the snail Lymnaea stagnalis. J Exp Biol 113:351-366.

Morton DW, Chiel HJ (1993a) In vivo buccal nerve activity that distinguishes ingestion from rejection can be used to predict behavioral transitions in Aplysia. J Comp Physiol [A] 172:17-32.

Morton DW, Chiel HJ (1993b) The timing of activity in motor neurons that produce radula movements distinguishes ingestion from rejection in Aplysia. J Comp Physiol [A] 173:519-536.

Mulloney B, Murchison D, Chrachri A (1993) Modular organization of pattern-generating circuits in a segmental motor system: the swimmerets of crayfish. Semin Neurosci 5:49-57.

Ono JK, McCaman RE (1980) Identification of additional histaminergic neurons in Aplysia: improvements of single cell isolation techniques for in tandem physiological and chemical studies. Neuroscience 5:835-840.

Perrins R, Roberts A (1995) Cholinergic contribution to excitation in a spinal locomotor central pattern generator in Xenopus embryos. J Neurophysiol 73:1013-1019.

Rao G, Barnes CA, McNaughton BL (1986) Intracellular fluorescent staining with carboxyfluorescein: a rapid and reliable method for quantifying dye-coupling in mammalian central nervous system. J Neurosci Methods 16:251-263.

Rosen SC, Weiss KR, Kupferman I (1979) Response properties and synaptic connections of mechanoafferent neurons in cerebral ganglion of Aplysia. J Neurophysiol 42:954-974.
Rosen SC, Weiss KR, Cohen JL, Kupfermann I (1982) Interganglionic cerebral-buccal mechanoafferents of Aplysia: receptive fields and synaptic connections to different classes of neurons involved in feeding behavior. J Neurophysiol 48:271-288.

Rosen SC, Weiss KR, Goldstein RS, Kupferman I (1989) The role of modulatory neuron in feeding and satiation in Aplysia: effects of lesioning the sertonergic metacerebral cells. J Neurosci 9:1562-1578.

Rosen SC, Halvorsen EL, Cropper EC, Miller MW, Weiss KR, Kupferman I (1990) Neuronal control of coordinated lip movements during feeding in Aplysia. Soc Neurosci Abstr 16:1227.

Rosen SC, Weiss KR, Kupferman I (1991a) Command of feeding in Aplysia: implication of a network of identified interganglionic interneurons in the buccal and cerebral ganglia. Soc Neurosci Abstr 17:595.6

Rosen SC, Teyke T, Miller MW, Weiss KR, Kupferman I (1991b) Identification and characterization of cerebral-to-buccal interneurons implicated in the control of motor programs associated with feeding in Aplysia. J Neurosci 11:3630-3655.

Rossignol S, Saltiel M-C, Perreault T, Drew K, Pearson K, Belanger M (1993) Intralimb and interlimb coordination in the cat during real and fictive rhythmic motor programs. Semin Neurosci 5:67-75.

Ryckebusch S, Laurent G (1994) Interactions between segmental leg central pattern generators during fictive rhythms in the locust. J Neurophysiol 72:2771-2785.

Sigvardt KA (1993) Intersegmental coordination in the lamprey central pattern generator for locomotion. Semin Neurosci 5:3-15.

Simmers AJ, Bush BMH (1983) Central nervous mechanisms controlling rhythmic burst generation in the ventilatory motoneurones of Carcinus maenus. J Comp Physiol [A] 150:1-21.

Susswein AJ, Byrne JH (1988) Identification and characterisation of neurons initiating patterned neural activity in the buccal ganglia of Aplysia. J Neurosci 8:2049-2061.

Teyke T, Rosen SC, Weiss KR, Kupferman I (1993) Dopaminergic neuron B20 generates rhythmic neuronal activity in the feeding motor circuitry of Aplysia. Brain Res 630:226-237.

Weiss KR, Cohen JL, Kupfermann I (1978) Modulatory control of buccal musculature by a serotonergic neuron (metacerebral cell) in Aplysia. J Neurophysiol 41:181-203.

Weiss KR, Koch UT, Koester J, Rosen SC, Kupferman I (1982) The role of arousal in modulating feeding behaviour of Aplysia: neural and behavioral studies. In: The neural basis of feeding and reward (Hoebel BG, Novin D, eds), pp 25-57. Brunswick, ME: Haer Institute.

Weiss KR, Chiel HJ, Koch U, Kupferman I (1986) Activity of an identified histaminergic neuron, and its possible role in arousal of feeding behavior in semi-intact Aplysia. J Neurosci 6:2403-2415.

Willows AOD (1980) Physiological basis of feeding behavior in Tritonia diomedea. II. Neuronal mechanisms. J Neurophysiol 44:849-861. 